\title{
Article
}

\section{Mixed-Norm Amalgam Spaces and Their Predual}

\author{
Houkun Zhang and Jiang Zhou *
}

Citation: Zhang, H.; Zhou, J. Mixed-Norm Amalgam Spaces and Their Predual. Symmetry 2022, 14, 74. https://doi.org/10.3390/sym14010074

Academic Editor: Ioan Rașa

Received: 10 November 2021 Accepted: 18 December 2021 Published: 4 January 2022

Publisher's Note: MDPI stays neutral with regard to jurisdictional claims in published maps and institutional affiliations.

Copyright: (c) 2022 by the authors. Licensee MDPI, Basel, Switzerland. This article is an open access article distributed under the terms and conditions of the Creative Commons Attribution (CC BY) license (https:// creativecommons.org/licenses/by/ $4.0 /)$.
College of Mathematics and System Sciences, Xinjiang University, Urumqi 830017, China; zhanghkmath@163.com or zhanghkmath@stu.xju.edu.cn

* Correspondence: zhoujiang@xju.edu.cn

\begin{abstract}
In this paper, we introduce mixed-norm amalgam spaces $\left(L^{\vec{p}}, L^{\vec{s}}\right)^{\alpha}\left(\mathbb{R}^{n}\right)$ and prove the boundedness of maximal function. Then, the dilation argument obtains the necessary and sufficient conditions of fractional integral operators' boundedness. Furthermore, the strong estimates of linear commutators $\left[b, I_{\gamma}\right]$ generated by $b \in B M O\left(\mathbb{R}^{n}\right)$ and $I_{\gamma}$ on mixed-norm amalgam spaces $\left(L^{\vec{p}}, L^{\vec{s}}\right)^{\alpha}\left(\mathbb{R}^{n}\right)$ are established as well. In order to obtain the necessary conditions of fractional integral commutators' boundedness, we introduce mixed-norm Wiener amalgam spaces $\left(L^{\vec{p}}, L^{\vec{s}}\right)\left(\mathbb{R}^{n}\right)$. We obtain the necessary and sufficient conditions of fractional integral commutators' boundedness by the duality theory. The necessary conditions of fractional integral commutators' boundedness are a new result even for the classical amalgam spaces. By the equivalent norm and the operators $S t_{r}^{(p)}(f)(x)$, we study the duality theory of mixed-norm amalgam spaces, which makes our proof easier. In particular, note that predual of the primal space is not obtained and the predual of the equivalent space does not mean the predual of the primal space.
\end{abstract}

Keywords: mixed norm; amalgam spaces; predual; fractional integral operators; commutators

\section{Introduction}

The fractional power of the Laplacian operators $\triangle$ are defined by

$$
\left((-\triangle)^{\gamma / 2}(f)\right)^{\wedge}(\xi)=(2 \pi|\xi|)^{\gamma} \hat{f}(\xi) .
$$

Comparing (1) to the Fourier transform of $|x|^{-\gamma}, 0<\gamma<n$, we are led to define the so-called fractional integral operators $I_{\gamma}$ by

$$
I_{\gamma} f(x)=(-\triangle)^{\gamma / 2}(f)(x)=C_{\gamma} \int_{\mathbb{R}^{n}} \frac{f(y)}{|x-y|^{n-\gamma}} d y,
$$

where

$$
C_{\gamma}^{-1}=\frac{\pi^{n / 2} 2^{\gamma} \Gamma(\gamma / 2)}{\Gamma((n-\gamma) / 2)}
$$

By simple calculation,

$$
\int_{\mathbb{R}^{n}} f(x) I_{\alpha} g(x) d x=\int_{\mathbb{R}^{n}} g(x) I_{\alpha} f(x) d x
$$

can be obtained. According to the symmetry, we can get the following result: if $I_{\alpha}$ are bounded from $X$ to $Y$, then $I_{\alpha}$ are also bounded from $Y^{\prime}$ to $X^{\prime}$, where $Y^{\prime}$ and $X^{\prime}$ are predual of $Y$ and $X$. An essential application of fractional integral operators' boundedness, via the well-known Hardy-Littlewood-Sobolev theorem, is proving the Sobolev embedding theorem. This paper investigates the generalization of the Hardy-Littlewood-Sobolev theorem on mixed-norm amalgam spaces.

Mixed-norm Lebesgue spaces, as natural generalizations of the classical Lebesgue spaces $L^{p}\left(\mathbb{R}^{n}\right)(0<p<\infty)$, were first introduced by Benedek and Panzone [1]. Due to 
the more precise structure of mixed-norm function spaces than the corresponding classical function spaces, mixed-norm function spaces have extensive applications in the partial differential equations [2-4]. So, the mixed-norm function spaces are widely introduced and studied, such as mixed-norm Lorentz spaces [5], mixed-norm Lorentz-Marcinkiewicz spaces [6], mixed-norm Orlicz spaces [7], anisotropic mixed-norm Hardy spaces [8], mixednorm Triebel-Lizorkin spaces [9], mixed Morrey spaces [10,11], and weak mixed-norm Lebesgue spaces [12]. More information can be found in [13].

The mixed-norm Lebesgue spaces are stated as follows. Let $f$ be a measurable function on $\mathbb{R}^{n}$ and $0<\vec{p} \leq \infty$. We say that $f$ belongs to the mixed-norm Lebesgue spaces $L^{\vec{p}}\left(\mathbb{R}^{n}\right)$, if the norm

$$
\|f\|_{L^{\vec{p}}}=\left(\int_{\mathbb{R}} \cdots\left(\int_{\mathbb{R}}\left|f\left(x_{1}, \cdots, x_{n}\right)\right|^{p_{1}} d x_{1}\right)^{\frac{p_{2}}{p_{1}}} \cdots d x_{n}\right)^{\frac{1}{p_{n}}}<\infty
$$

with the usual modification when $p_{i}=\infty$. Note that if $p_{1}=p_{2}=\cdots=p_{n}=p$, then $L^{\vec{p}}\left(\mathbb{R}^{n}\right)$ are reduced to classical Lebesgue spaces $L^{p}$ and

$$
\|f\|_{L^{\vec{p}}}=\|f\|_{L^{p}}=\left(\int_{\mathbb{R}^{n}}|f(x)|^{p} d x\right)^{\frac{1}{p}}
$$

with the usual modification when $p_{i}=\infty$. Furthermore, let $\left\{q_{1}, \cdots, q_{n}\right\}$ and $\left\{s_{1}, \cdots, s_{n}\right\}$ be rearrangements of the set $\left\{p_{1}, \cdots, p_{n}\right\}$, and satisfy

$$
q_{1} \leq q_{2} \leq \cdots \leq q_{n} \text { and } s_{1} \geq s_{2} \geq \cdots \geq s_{n}
$$

Then, by Minkowski's inequality,

$$
\|f\|_{L_{\vec{q}}} \leq\|f\|_{L_{\vec{p}}} \leq\|f\|_{L^{\vec{s}}}
$$

where $\vec{q}=\left(q_{1}, \cdots, q_{n}\right), \vec{s}=\left(s_{1}, \cdots, s_{n}\right)$, and $\vec{p}=\left(p_{1}, \cdots, p_{n}\right)$.

In partial differential equations, Morrey spaces $\mathcal{M}_{p, \lambda}\left(\mathbb{R}^{n}\right)$, introduced by Morrey in 1938 [14], are widely used to investigate the local behavior of solutions to elliptic and parabolic differential equations. These spaces were defined as follows. For $0 \leq \lambda \leq n, 1 \leq$ $p \leq \infty$, we say that $f \in \mathcal{M}_{p, \lambda}\left(\mathbb{R}^{n}\right)$ if $f \in L_{1}^{\text {loc }}\left(\mathbb{R}^{n}\right)$ and

$$
\begin{aligned}
\|f\|_{\mathcal{M}_{p, \lambda}} & =\sup _{x \in \mathbb{R}^{n}} \sup _{r>0} r^{-\frac{\lambda}{p}}\|f\|_{L_{p}(Q(x, r))} \\
& =\sup _{r>0} \sup _{x \in \mathbb{R}^{n}} r^{-\frac{\lambda}{p}}\|f\|_{L_{p}(Q(x, r))}<\infty .
\end{aligned}
$$

It is obvious that if $\lambda=0$, then $\mathcal{M}_{p, 0}\left(\mathbb{R}^{n}\right)=L_{p}\left(\mathbb{R}^{n}\right)$; if $\lambda=n$, then $\mathcal{M}_{p, n}\left(\mathbb{R}^{n}\right)=$ $L_{\infty}\left(\mathbb{R}^{n}\right)$; if $\lambda<0$ or $\lambda>n$, then $\mathcal{M}_{p, \lambda}=\Theta$, where $\Theta$ is the set of all functions almost everywhere equivalent to 0 on $\mathbb{R}^{n}$.

Moreover, combining mixed Lebesgue spaces and Morrey spaces, Nogayama, in 2019, introduced mixed Morrey spaces [10,11]. Mixed Morrey spaces were stated as follows. Let $\vec{p}=\left(p_{1}, p_{2}, \cdots, p_{n}\right) \in(0, \infty]^{n}$ and $p_{0} \in(0, \infty]$ satisfy

$$
\sum_{j=1}^{n} \frac{1}{p_{j}} \geq \frac{n}{p_{0}} .
$$


The mixed Morrey spaces $\mathcal{M}_{\vec{p}}^{p_{0}}\left(\mathbb{R}^{n}\right)$ were defined to be the set of all measurable functions $f$ such that their quasi-norms

$$
\begin{aligned}
\|f\|_{\mathcal{M}_{\vec{p}}^{p_{0}}} & =\sup _{x \in \mathbb{R}^{n}} \sup _{r>0}|Q(x, r)|^{\frac{1}{p_{0}}-\frac{1}{n} \sum_{j=1}^{n} \frac{1}{p_{j}}}\left\|f \chi_{Q(x, r)}\right\|_{L_{\vec{p}}} \\
& =\sup _{r>0} \sup _{x \in \mathbb{R}^{n}}|Q(x, r)|^{\frac{1}{p_{0}}-\frac{1}{n} \sum_{j=1}^{n} \frac{1}{p_{j}}}\left\|f \chi_{Q(x, r)}\right\|_{L_{\vec{p}}} \\
& <\infty
\end{aligned}
$$

are finite. It is obvious that $\mathcal{M}_{\vec{p}}^{p_{0}}\left(\mathbb{R}^{n}\right)=\mathcal{M}_{p}^{p_{0}}\left(\mathbb{R}^{n}\right)=\mathcal{M}_{p, \lambda}\left(\mathbb{R}^{n}\right)$ if $p_{1}=p_{2}=\cdots=p_{n}=p$ and $\mathcal{M}_{\vec{p}}^{p_{0}}\left(\mathbb{R}^{n}\right)=L_{\vec{p}}\left(\mathbb{R}^{n}\right)$ if $\frac{1}{p_{0}}=\frac{1}{n} \sum_{j=1}^{n} \frac{1}{p_{j}}$.

Inspired by global Morrey-type spaces $G M_{p \theta, \omega}\left(\mathbb{R}^{n}\right)$ [15] and mixed Morrey spaces, the global mixed Morrey-type spaces $G M_{\vec{p} \theta, \omega}\left(\mathbb{R}^{n}\right)$ were defined [16] as follows. For any functions $f \in L_{1}^{\operatorname{loc}}\left(\mathbb{R}^{n}\right)$, we say $f \in L M_{\vec{p} \theta, \omega}\left(\mathbb{R}^{n}\right)$ when the quasi-norms

$$
\begin{aligned}
\|f\|_{G M_{\vec{p} \theta, \omega}} & =\sup _{x \in \mathbb{R}^{n}}\|\omega(r)\| f \chi_{Q(x, r)}\left\|_{L_{\vec{p}}}\right\|_{L_{\theta}(0, \infty)} \\
& =\sup _{x \in \mathbb{R}^{n}}\left(\int_{0}^{\infty}\left|\omega(r)\left\|f \chi_{Q(x, r)}\right\|_{L_{\vec{p}}}\right|^{\theta} d r\right)^{\frac{1}{\theta}}<\infty .
\end{aligned}
$$

The global mixed Morrey-type spaces can be regarded as mixed Morrey spaces by replacing the $L^{\infty}$-norm for $r$ by the $L^{\theta}$-norm. Due to symmetry, the interesting mixed-norm amalgam spaces $\left(L^{\vec{p}}, L^{\vec{s}}\right)^{\alpha}\left(\mathbb{R}^{n}\right)$ are introduced, which can be regarded as mixed Morrey spaces by replacing the $L^{\infty}$-norm for $x$ by the $L^{\vec{s}}$-norm. In order to study $\left(L^{\vec{p}}, L^{\vec{s}}\right)^{\alpha}\left(\mathbb{R}^{n}\right)$, a kind of mixed-norm Wiener amalgam space $\left(L^{\vec{p}}, L^{\vec{s}}\right)\left(\mathbb{R}^{n}\right)$ was also introduced. In particular, Zhao et al. first introduced mixed-norm Wiener amalgam spaces [17] which are different from mixed-norm Wiener amalgam spaces in this paper. Furthermore, according to Proposition 5, the mixed-norm amalgam spaces $\left(L^{\vec{p}}, L^{\vec{s}}\right)^{\alpha}\left(\mathbb{R}^{n}\right)$ and $\left(L^{\vec{p}}, L^{\vec{s}}\right)\left(\mathbb{R}^{n}\right)$ can also be seen as the generalizations of the classical amalgam spaces $\left(L^{p}, \ell^{s}\right)^{\alpha}\left(\mathbb{R}^{n}\right)$ and $\left(L^{p}, \ell^{s}\right)\left(\mathbb{R}^{n}\right)$. Let us recall some information on classical amalgam spaces.

The amalgam spaces $\left(L^{p}, \ell^{s}\right)\left(\mathbb{R}^{n}\right)$ were first introduced by Wiener [18] in 1926. However, their systematic study goes back to the works of Holland [19], who studied the Fourier transform on $\mathbb{R}^{n}$. Besides that, the spaces have been widely studied [20-23]. It is obvious that Lebesgue space $L^{p}\left(\mathbb{R}^{n}\right)$ coincides with the amalgam space $\left(L^{p}, \ell^{p}\right)\left(\mathbb{R}^{n}\right)$. For any $r>0$, the dilation operator $S t_{r}^{(p)}: f(x) \mapsto r^{-\frac{n}{p}} f\left(r^{-1} x\right)$ is isometric on $L^{p}\left(\mathbb{R}^{n}\right)$. However, amalgam spaces do not have this property. If $p \neq s$, there does not exist $\alpha$ such that $\sup _{r>0}\left\|S t_{r}^{(\alpha)}(f)\right\|_{\left(L^{p}, \ell^{s}\right)}<\infty$, although $S t_{r}^{(\alpha)}(f) \in\left(L^{p}, \ell^{s}\right)\left(\mathbb{R}^{n}\right)$ for all $f \in\left(L^{p}, \ell^{s}\right)\left(\mathbb{R}^{n}\right)$, $\rho>0$ and $\alpha>0$ [24]. The amalgam spaces $\left(L^{p}, \ell^{s}\right)^{\alpha}\left(\mathbb{R}^{n}\right)$ compensate this shortcoming. The functions spaces $\left(L^{p}, \ell^{s}\right)^{\alpha}\left(\mathbb{R}^{n}\right)$ were introduced by Fofana in 1988 [25], which consist of $f \in\left(L^{p}, \ell^{s}\right)\left(\mathbb{R}^{n}\right)$ and satisfy $\sup _{r>0}\left\|S t_{r}^{(\alpha)}(f)\right\|_{\left(L^{p}, \ell^{s}\right)}<\infty$. Finally, we point out that many new amalgam spaces have been introduced, such as variable exponent amalgam spaces $\left(L^{p(x)}, L^{s(x)}\right)\left(\mathbb{R}^{n}\right)[26]$ and Orlicz amalgam spaces $\left(L_{t}^{\Phi}, \ell^{s}\right)\left(\mathbb{R}^{n}\right)[27]$.

Various mixed-norm function spaces have shown the boundedness properties of $I_{\gamma}$ extensively. In 1960, Benedek and Panzone first studied the boundedness of $I_{\gamma}$ from mixed-norm Lebesgue spaces $L^{\vec{p}}\left(\mathbb{R}^{n}\right)$ to mixed-norm Lebesgue spaces $L^{\vec{q}}\left(\mathbb{R}^{n}\right)$ [1], which is a generalization of the classical Hardy-Littlewood-Sobolev theorem (see [28]). In 2021, Zhang and Zhou improved the theorem on mixed-norm Lebesgue spaces, which is stated as follows.

Lemma 1 (see [16]). Let $0<\gamma<n$ and $1<\vec{p}, \vec{q}<\infty$. Then,

$$
1<\vec{p} \leq \vec{q}<\infty, \gamma=\sum_{i=1}^{n} \frac{1}{p_{i}}-\sum_{i=1}^{n} \frac{1}{q_{i}}
$$


if and only if

$$
\left\|I_{\gamma} f\right\|_{L_{\vec{q}}} \lesssim\|f\|_{L_{\vec{p}}}
$$

For a locally integrable function $b$, the commutators of fractional integral operators $I_{\gamma}$ are defined by

$$
\left[b, I_{\gamma}\right] f(x):=b(x) I_{\gamma} f(x)-I_{\gamma}(b f)(x)=C_{\gamma} \int_{\mathbb{R}^{n}} \frac{(b(x)-b(y)) f(y)}{|x-y|^{n-\gamma}} d y,
$$

which were introduced by Chanillo in [29]. These commutators can also be used to study the theory of Hardy spaces $H^{p}\left(\mathbb{R}^{n}\right)$ [30]. In 2019, Nogayama gave an characterization of $B M O\left(\mathbb{R}^{n}\right)$ spaces via the $\left(\mathcal{M}_{\vec{p}}^{p_{0}}, \mathcal{M}_{\vec{q}}^{q_{0}}\right)$-boundedness of $\left[b, I_{\gamma}\right]$ [11]. In 2021, the result was improved on mixed-norm Lebesgue in [31], which is stated as follows.

Lemma 2 (see [31]). Let $0<\gamma<n, 1<\vec{p} \leq \vec{q}<\infty$ and

$$
\gamma=\sum_{i=1}^{n} \frac{1}{p_{i}}-\sum_{i=1}^{n} \frac{1}{q_{i}}
$$

Then, the following conditions are equivalent:

(i) $\quad b \in B M O\left(\mathbb{R}^{n}\right)$.

(ii) $\left[b, I_{\gamma}\right]$ is bounded from $L^{\vec{p}}\left(\mathbb{R}^{n}\right)$ to $L^{\vec{q}}\left(\mathbb{R}^{n}\right)$.

Similar to symmetry of $I_{\alpha}$, we have

$$
\int_{\mathbb{R}^{n}} f(x)\left[b, I_{\alpha}\right] g(x) d x=-\int_{\mathbb{R}^{n}} g(x)\left[b, I_{\alpha}\right] f(x) d x,
$$

and if $I_{\alpha}$ are bounded from $X$ to $Y$, then $\left[b, I_{\alpha}\right]$ are also bounded from $Y^{\prime}$ to $X^{\prime}$, where $Y^{\prime}$ and $X^{\prime}$ are predual of $Y$ and $X$.

In addition, we point out that the boundedness of fractional integral operators and their commutators have been studied in classical amalgam spaces. In 2020, Wang showed the boundedness of fractional integral operators $I_{\gamma}$ and their commutators from $\left(L^{p}, L^{s}\right)^{\alpha}\left(\mathbb{R}^{n}\right)$ to $\left(L^{q}, L^{s}\right)^{\alpha}\left(\mathbb{R}^{n}\right)$ with $1 / p-1 / q=1 / \alpha-1 / \beta=\gamma / n$ [32]. Nevertheless, Wang does not prove the necessary conditions of fractional integral operators and their commutators. Using the dilation argument, we obtain the necessary and sufficient conditions of fractional integral operators in this paper. We obtain the necessary and sufficient conditions of fractional integral commutators' boundedness by the duality theory.

Now, let us recall that the definition of $B M O\left(\mathbb{R}^{n}\right) . B M O\left(\mathbb{R}^{n}\right)$ is the Banach function space modulo constants with the norm $\|\cdot\|_{B M O}$ defined by

$$
\|b\|_{B M O}=\sup _{B \subset \mathbb{R}^{n}} \frac{1}{|B|} \int_{B}\left|b(y)-b_{B}\right| d y<\infty,
$$

where the supremum is taken over all balls $B$ in $\mathbb{R}^{n}$ and $b_{B}$ stands for the mean value of $b$ over $B$; that is, $b_{B}:=(1 /|B|) \int_{B} b(y) d y$. By John-Nirenberg inequality,

$$
\|b\|_{B M O} \sim \sup _{B \subset \mathbb{R}^{n}} \frac{\left\|b-b_{B}\right\|_{L^{p}}}{\left\|\chi_{B}\right\|_{L^{p}}}, 1<p<\infty .
$$

It is also right if we replace the $L^{p}$-norm by mixed-norm $L^{\vec{p}}$-norm (see Lemma 5).

We first define mixed-norm amalgam spaces, which extend classical amalgam spaces and mixed Morrey spaces. Studying the boundedness of $I_{\gamma}$ and $\left[b, I_{\gamma}\right]$ in these new spaces is natural and important. Before that, we also studied some properties of the new spaces. This paper is organized as follows. In Section 2, we state definitions of mixed-norm amalgam spaces, some properties of mixed-norm amalgam spaces, and the main results of the present 
paper. We give the proof of some properties of mixed-norm amalgam spaces in Section 3. We investigate the predual of mixed-norm amalgam spaces in Section 4. In Section 5, the boundedness of maximal function on mixed-norm amalgam spaces $\left(L^{\vec{p}}, L^{\vec{s}}\right)^{\alpha}\left(\mathbb{R}^{n}\right)$ is investigated as well as the rationality of fractional integral operators. In Sections 6 and 7 , we prove the boundedness of $I_{\gamma}$ and their commutators generated by $b \in B M O\left(\mathbb{R}^{n}\right)$. In the final section, we study the necessary condition of the boundedness of $\left[b, I_{\gamma}\right]$ from $\left(L^{\vec{p}}, L^{\vec{s}}\right)^{\alpha}\left(\mathbb{R}^{n}\right)$ to $\left(L^{\vec{q}}, L^{\vec{s}}\right)^{\beta}\left(\mathbb{R}^{n}\right)$, which is a new result even for the classical amalgam spaces.

Next, we make some conventions and recall some notions. Let $\vec{p}=\left(p_{1}, p_{2}, \cdots, p_{n}\right)$, $\vec{q}=\left(q_{1}, q_{2}, \cdots, q_{n}\right), \vec{s}=\left(s_{1}, s_{2}, \cdots, s_{n}\right)$ be n-tuples and $1<p_{i}, q_{i}, s_{i}<\infty, i=1,2, \cdots, n$. We define that if $\varphi(a, b)$ is a relation or equation among numbers, $\varphi(\vec{p}, \vec{q})$ will mean that $\varphi\left(p_{i}, q_{i}\right)$ holds for each $i$. For example, $\vec{p}<\vec{q}$ means that $p_{i}<q_{i}$ holds for each $i$ and $\frac{1}{\bar{p}}+\frac{1}{\vec{p}^{\prime}}=1$ means $\frac{1}{p_{i}}+\frac{1}{p_{i}^{\prime}}=1$ holds for each $i$. The symbol $B$ denotes the open ball and $B(x, r)$ denotes the open ball centered at $x$ of radius $r$. Let $\rho B(x, r)=B(x, \rho r)$, where $\rho>0$. $A \sim B$ means that $A$ is equivalent to $B$, that is, $A \lesssim B(A \leq C B)$ and $B \lesssim A(B \leq C A)$, where $C$ is a positive constant. Throughout the paper, each positive constant $C$ is not necessarily equal.

\section{Mixed-Norm Amalgam Spaces $\left(L^{\vec{p}}, L^{\vec{s}}\right)\left(\mathbb{R}^{n}\right)$ and $\left(L^{\vec{p}}, L^{\vec{s}}\right)^{\alpha}\left(\mathbb{R}^{n}\right)$}

The definitions of mixed-norm amalgam spaces and some properties of mixed-norm amalgam spaces are presented in Section 2.1. Then, the main theorems are shown in Section 2.2.

\subsection{Definitions and Properties}

In this section, we present the definitions of mixed-norm amalgam spaces $\left(L^{\vec{p}}, L^{\vec{s}}\right)\left(\mathbb{R}^{n}\right)$ and $\left(L^{\vec{p}}, L^{\vec{s}}\right)^{\alpha}\left(\mathbb{R}^{n}\right)$ and their properties. Firstly, the definitions of mixed-norm amalgam spaces $\left(L^{\vec{p}}, L^{\vec{s}}\right)\left(\mathbb{R}^{n}\right)$ and $\left(L^{\vec{p}}, L^{\vec{s}}\right)^{\alpha}\left(\mathbb{R}^{n}\right)$ are given as follows.

Definition 1. Let $1 \leq \vec{p}, \vec{s}, \alpha \leq \infty$. We define two types of amalgam spaces of $L^{\vec{p}}\left(\mathbb{R}^{n}\right)$ and $L^{\vec{s}}\left(\mathbb{R}^{n}\right)$. If measurable functions $f$ satisfy $f \in L_{l o c}^{1}\left(\mathbb{R}^{n}\right)$, then

$$
\left(L^{\vec{p}}, L^{\vec{s}}\right)\left(\mathbb{R}^{n}\right):=\left\{f:\|f\|_{\left(L^{\vec{p}}, L^{\vec{s}}\right)}<\infty\right\}
$$

and

$$
\left(L^{\vec{p}}, L^{\vec{s}}\right)^{\alpha}\left(\mathbb{R}^{n}\right):=\left\{f:\|f\|_{\left(L^{\vec{p}}, L^{\vec{s}}\right)^{\alpha}}<\infty\right\}
$$

where

$$
\|f\|_{\left(L^{\vec{p}}, L^{\vec{s}}\right)}=\|\| f \chi_{B(\cdot, 1)}\left\|_{L^{\vec{p}}}\right\|_{L^{\vec{s}}}=\left(\int_{\mathbb{R}} \cdots\left(\int_{\mathbb{R}}\left\|f \chi_{B(y, 1)}\right\|_{L^{\vec{p}}}^{s_{1}} d y_{1}\right)^{\frac{s_{2}}{s_{1}}} \cdots d y_{n}\right)^{\frac{1}{s_{n}}}
$$

and

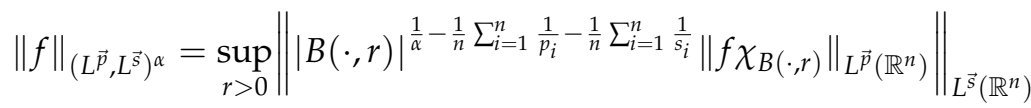

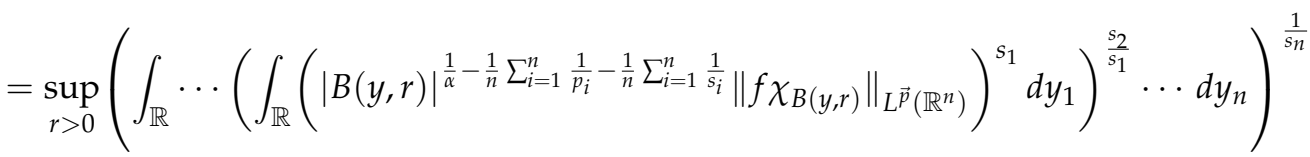

with the usual modification for $p_{i}=\infty$ or $s_{i}=\infty$.

Remark 1. By Definition 1, we have

(i) If $p_{i}=p$ and $s_{i}=s$ for each $i$, then

$$
\left(L^{\vec{p}}, L^{\vec{s}}\right)\left(\mathbb{R}^{n}\right)=\left(L^{p}, L^{s}\right)\left(\mathbb{R}^{n}\right), \quad\left(L^{\vec{p}}, L^{\vec{s}}\right)^{\alpha}\left(\mathbb{R}^{n}\right)=\left(L^{p}, L^{s}\right)^{\alpha}\left(\mathbb{R}^{n}\right) ;
$$


(ii) If $s_{i}=\infty$ for each $i$ and $\frac{1}{\alpha} \leq \frac{1}{n} \sum_{i=1}^{n} \frac{1}{p_{i}}$, then

$$
\left(L^{\vec{p}}, L^{\vec{s}}\right)^{\alpha}\left(\mathbb{R}^{n}\right)=\mathcal{M}_{\vec{p}}^{\alpha}\left(\mathbb{R}^{n}\right),
$$

where $\mathcal{M}_{\vec{p}}^{\alpha}\left(\mathbb{R}^{n}\right)$ is mixed Morrey spaces defined as $[10,11]$. In particular, the condition $\frac{1}{\alpha} \leq \frac{1}{n} \sum_{i=1}^{n} \frac{1}{p_{i}}$ inspired us to study Proposition 2 .

Next, we claim that the mixed-norm amalgam spaces defined in Definition 1 are Banach spaces.

Proposition 1. Let $1 \leq \vec{p}, \vec{s}, \alpha \leq \infty$. Mixed norm amalgam spaces $\left(L^{\vec{p}}, L^{\vec{s}}\right)\left(\mathbb{R}^{n}\right)$ and $\left(L^{\vec{p}}, L^{\vec{s}}\right)^{\alpha}\left(\mathbb{R}^{n}\right)$ are also Banach spaces.

The following proposition shows the necessary relationship of the index $\vec{p}, \vec{s}$ and $\alpha$.

Proposition 2. The spaces $\left(L^{\vec{p}}, L^{\vec{s}}\right)^{\alpha}\left(\mathbb{R}^{n}\right)$ are nontrivial if and only if $\frac{1}{n} \sum_{i=1}^{n} \frac{1}{s_{i}} \leq \frac{1}{\alpha} \leq \frac{1}{n} \sum_{i=1}^{n} \frac{1}{p_{i}}$.

Some embedding results are shown as follows.

Proposition 3. Let $1 \leq \vec{p}, \vec{q}, \vec{s} \leq \infty, \frac{1}{n} \sum_{i=1}^{n} \frac{1}{s_{i}} \leq \frac{1}{\alpha} \leq \frac{1}{n} \sum_{i=1}^{n} \frac{1}{p_{i}}$, and $\frac{1}{n} \sum_{i=1}^{n} \frac{1}{s_{i}} \leq \frac{1}{\alpha} \leq$ $\frac{1}{n} \sum_{i=1}^{n} \frac{1}{q_{i}}$. Then,

(i) $\quad\left(L^{\vec{p}}, L^{\vec{s}}\right)^{\alpha}\left(\mathbb{R}^{n}\right) \subset\left(L^{\vec{p}}, L^{\vec{s}}\right)\left(\mathbb{R}^{n}\right)$ with $\|f\|_{\left(L^{\vec{p}}, L^{\vec{s}}\right)} \leq\|f\|_{\left(L^{\vec{p}}, L^{\vec{s}}\right)^{\alpha}}$;

(ii) If $\vec{p} \leq \vec{q},\left(L^{\vec{q}}, L^{\vec{s}}\right)^{\alpha}\left(\mathbb{R}^{n}\right) \subseteq\left(L^{\vec{p}}, L^{\vec{s}}\right)^{\alpha}\left(\mathbb{R}^{n}\right)$ with $\|f\|_{\left(L^{\vec{p}}, L^{\vec{s}}\right)^{\alpha}} \leq\|f\|_{\left(L^{\vec{q}}, L^{\vec{s}}\right)^{\alpha}}$.

We give an estimate of characteristic function on $\left(L^{\vec{p}}, L^{\vec{s}}\right)^{\alpha}\left(\mathbb{R}^{n}\right)$ and $\mathcal{H}\left(\vec{p}^{\prime}, \vec{s}^{\prime}, \alpha^{\prime}\right)\left(\mathbb{R}^{n}\right)$ as follows.

Proposition 4. Let $0 \leq \frac{1}{n} \sum_{i=1}^{n} \frac{1}{s_{i}} \leq \frac{1}{\alpha} \leq \frac{1}{n} \sum_{i=1}^{n} \frac{1}{p_{i}}<1$ and $\chi_{B\left(x_{0}, r_{0}\right)}$ is a characteristic function on $B\left(x_{0}, r_{0}\right)$. Then, we have

$$
\left\|\chi_{B\left(x_{0}, r_{0}\right)}\right\|_{\left(L^{\vec{p}}, L^{\vec{s}}\right)^{\alpha}} \lesssim r_{0}^{n / \alpha} \text { and }\left\|\chi_{B\left(x_{0}, r_{0}\right)}\right\|_{\mathcal{H}\left(\vec{p}^{\prime}, \vec{s}^{\prime}, \alpha^{\prime}\right)} \lesssim r_{0}^{n / \alpha^{\prime}}
$$

\subsection{Main Theorems}

In this section, we show the main theorems in this paper. First, we define two types "discrete" mixed-norm amalgam spaces which are equivalent to mixed-norm amalgam spaces in Definition 1. Let $Q_{r, k}=r\left[k+[0,1)^{n}\right]$ and

$$
\left\|\left\{a_{k}\right\}_{k \in \mathbb{Z}^{n}}\right\|_{\ell^{s}}:=\left(\sum_{k_{n} \in \mathbb{Z}} \cdots\left(\sum_{k_{1} \in \mathbb{Z}}\left|a_{k}\right|^{s_{1}}\right)^{\frac{s_{2}}{s_{1}}} \cdots\right)^{\frac{1}{s_{n}}}
$$

with the usual modification for $s_{i}=\infty$.

Proposition 5. Let $1 \leq \vec{p}, \vec{s}, \alpha \leq \infty$ and $\frac{1}{n} \sum_{i=1}^{n} \frac{1}{s_{i}} \leq \frac{1}{\alpha} \leq \frac{1}{n} \sum_{i=1}^{n} \frac{1}{p_{i}}$. We define two types "discrete" mixed-norm amalgam spaces.

$$
\left(L^{\vec{p}}, \ell^{\vec{s}}\right)\left(\mathbb{R}^{n}\right):=\left\{f \in L_{l o c}^{1}:\|f\|_{\vec{p}, \vec{s}}:=\left\|\left\{\left\|f \chi_{Q_{1, k}}\right\|_{\vec{p}}\right\}_{k \in \mathbb{Z}^{n}}\right\|_{\ell^{\vec{s}}}<\infty\right\}
$$

and

$$
\left(L^{\vec{p}}, \ell^{\vec{s}}\right)^{\alpha}\left(\mathbb{R}^{n}\right):=\left\{f \in L_{l o c}^{1}:\|f\|_{\vec{p}, \vec{s}, \alpha}:=\sup _{r>0} r^{\frac{n}{\alpha}-\sum_{i=1}^{n} \frac{1}{p_{i}}} r\|f\|_{\vec{p}, \vec{s}}<\infty\right\},
$$


where

$$
r\|f\|_{\vec{p}, \vec{s}}:=\left\|\left\{\left\|f \chi_{Q_{r, k}}\right\|_{\vec{p}}\right\}_{k \in \mathbb{Z}^{n}}\right\|_{\ell^{\vec{s}}} .
$$

In fact, we have

$$
\left(L^{\vec{p}}, L^{\vec{s}}\right)\left(\mathbb{R}^{n}\right)=\left(L^{\vec{p}}, \ell^{\vec{s}}\right)\left(\mathbb{R}^{n}\right) \text { and }\left(L^{\vec{p}}, L^{\vec{s}}\right)^{\alpha}\left(\mathbb{R}^{n}\right)=\left(L^{\vec{p}}, \ell^{\vec{s}}\right)^{\alpha}\left(\mathbb{R}^{n}\right) .
$$

According to Proposition 5, we give the definition of the predual of mixed-norm amalgam spaces $\left(L^{\vec{p}}, \ell^{\vec{s}}\right)^{\alpha}\left(\mathbb{R}^{n}\right)$.

Definition 2. Let $1 \leq \vec{p}, \vec{s}, \alpha \leq \infty$ and $\frac{1}{n} \sum_{i=1}^{n} \frac{1}{s_{i}} \leq \frac{1}{\alpha} \leq \frac{1}{n} \sum_{i=1}^{n} \frac{1}{p_{i}}$. The space $\mathcal{H}\left(\vec{p}^{\prime}, \vec{s}^{\prime}, \alpha^{\prime}\right)$ is defined as the set of all elements of $L_{\text {loc }}^{1}\left(\mathbb{R}^{n}\right)$ for which there exists a sequence $\left\{\left(c_{j}, r_{j}, f_{j}\right)\right\}_{j \geq 1}$ of elements of $\mathbb{C} \times(0, \infty) \times\left(L^{\vec{p}^{\prime}}, \ell^{\vec{s}^{\prime}}\right)\left(\mathbb{R}^{n}\right)$ such that

$$
\begin{gathered}
f:=\sum_{j \geq 1} c_{j} S t_{r_{j}}^{\left(\alpha^{\prime}\right)}\left(f_{j}\right) \text { in the sense of } L_{l o c}^{1}\left(\mathbb{R}^{n}\right) ; \\
\left\|f_{j}\right\|_{\vec{p}^{\prime}, \vec{s}^{\prime}} \leq 1, j \geq 1 ; \\
\sum_{j \leq 1}\left|c_{j}\right|<\infty .
\end{gathered}
$$

We will always refer to any sequence $\left\{\left(c_{j}, r_{j}, f_{j}\right)\right\}_{j \geq 1}$ of elements of $\mathbb{C} \times(0, \infty) \times$ $\left(L^{\vec{p}^{\prime}}, \ell^{\vec{s}^{\prime}}\right)\left(\mathbb{R}^{n}\right)$ satisfying (2)-(4) as a block decomposition of $f$. For any element $f$ of $\mathcal{H}\left(\vec{p}^{\prime}, \vec{s}^{\prime}, \alpha^{\prime}\right)$, we set

$$
\|f\|_{\mathcal{H}\left(\vec{p}^{\prime}, \vec{s}^{\prime}, \alpha^{\prime}\right)}:=\inf \left\{\sum_{j \geq 1}\left|c_{j}\right|: f:=\sum_{j \geq 1} c_{j} S t_{r_{j}}^{\left(\alpha^{\prime}\right)} f_{j}\right\},
$$

where the infimum is taken over all block decompositions of $f$.

Theorem 1. (i) Let $1 \leq \vec{p}, \vec{s} \leq \infty$, and $\frac{1}{n} \sum_{i=1}^{n} \frac{1}{s_{i}} \leq \frac{1}{\alpha} \leq \frac{1}{n} \sum_{i=1}^{n} \frac{1}{p_{i}}$. If $g \in\left(L^{\vec{p}}, \ell^{\vec{s}}\right)^{\alpha}$ and $f \in \mathcal{H}\left(\vec{p}^{\prime}, \vec{s}^{\prime}, \alpha^{\prime}\right)$, we obtain $f g \in L^{1}\left(\mathbb{R}^{n}\right)$ and

$$
\left|\int_{\mathbb{R}^{n}} f(x) g(x) d x\right| \leq\|g\|_{\left(L^{\vec{p}}, \ell^{\vec{s}}\right)^{\alpha}}\|f\|_{\mathcal{H}\left(\vec{p}^{\prime}, \vec{s}^{\prime}, \alpha^{\prime}\right)} .
$$

(ii) Let $1<\vec{p}, \vec{s} \leq \infty$ and $\frac{1}{n} \sum_{i=1}^{n} \frac{1}{s_{i}} \leq \frac{1}{\alpha} \leq \frac{1}{n} \sum_{i=1}^{n} \frac{1}{p_{i}}$. The operator $T: g \mapsto T_{g}$ defined as

$$
<T_{g}, f>=\int_{\mathbb{R}^{n}} f(x) g(x) d x, g \in\left(L^{\vec{p}}, \ell^{\vec{s}}\right)^{\alpha}\left(\mathbb{R}^{n}\right) \text { and } f \in \mathcal{H}\left(\vec{p}^{\prime}, \vec{s}^{\prime}, \alpha^{\prime}\right)
$$

is an isometric isomorphism of $\left(L^{\vec{p}}, \ell^{\vec{s}}\right)^{\alpha}\left(\mathbb{R}^{n}\right)$ into $\mathcal{H}\left(\vec{p}^{\prime}, \vec{s}^{\prime}, \alpha^{\prime}\right)^{*}$.

(iii) Symmetric with

$$
\|f\|_{\left(L^{\vec{p}}, \ell^{\vec{s}}\right)^{\alpha}}=\sup \left\{\int_{\mathbb{R}^{n}}|f(x) g(x)| d x: g \in \mathcal{H}\left(\vec{p}^{\prime}, \vec{s}^{\prime}, \alpha^{\prime}\right),\|g\|_{\mathcal{H}\left(\vec{p}^{\prime}, \vec{s}^{\prime}, \alpha^{\prime}\right)} \leq 1\right\},
$$

we have

$$
\|f\|_{\mathcal{H}\left(\vec{p}^{\prime}, \vec{s}^{\prime}, \alpha^{\prime}\right)}=\sup \left\{\int_{\mathbb{R}^{n}}|f(x) g(x)| d x: g \in\left(L^{\vec{p}}, \ell^{\vec{s}}\right)^{\alpha}\left(\mathbb{R}^{n}\right),\|g\|_{\left(L^{\vec{p}}, l^{\vec{l}}\right)^{\alpha}} \leq 1\right\} .
$$

Before studying fractional integrals, we give the boundedness of maximal function, which shows the rationality of fractional integral operators on mixed-norm amalgam spaces $\left(L^{\vec{p}}, L^{\vec{s}}\right)^{\alpha}\left(\mathbb{R}^{n}\right)$. 
Theorem 2. Let $0<\gamma<n, 1<\vec{p}, \vec{s} \leq \infty, \frac{1}{n} \sum_{i=1}^{n} \frac{1}{s_{i}} \leq \frac{1}{\alpha} \leq \frac{1}{n} \sum_{i=1}^{n} \frac{1}{p_{i}}$. Then, the maximal function $M$ is bounded on $\left(L^{\vec{p}}, L^{\vec{s}}\right)^{\alpha}\left(\mathbb{R}^{n}\right)$. The maximal function $M$ is defined as

$$
M f(x)=\sup _{B \ni x} \frac{1}{|B|} \int_{B}|f(y)| d y,
$$

where the supremum is taken over all balls $B \subset \mathbb{R}^{n}$ containing $x$.

Now, we show the boundedness of fractional integral operators on mixed-norm amalgam spaces.

Theorem 3. Let $0<\gamma<n, 1<\vec{p} \leq \vec{q}<\infty, 1<\vec{s} \leq \infty, \frac{1}{n} \sum_{i=1}^{n} \frac{1}{s_{i}} \leq \frac{1}{\alpha} \leq \frac{1}{n} \sum_{i=1}^{n} \frac{1}{p_{i}}$, and $\frac{1}{n} \sum_{i=1}^{n} \frac{1}{s_{i}} \leq \frac{1}{\beta} \leq \frac{1}{n} \sum_{i=1}^{n} \frac{1}{q_{i}}$. Assume that $\gamma=\sum_{i=1}^{n} \frac{1}{p_{i}}-\sum_{i=1}^{n} \frac{1}{q_{i}}$. Then, the fractional integral operators $I_{\gamma}$ are bounded from $\left(L^{\vec{p}}, L^{\vec{s}}\right)^{\alpha}\left(\mathbb{R}^{n}\right)$ to $\left(L^{\vec{q}}, L^{\vec{s}}\right)^{\beta}\left(\mathbb{R}^{n}\right)$ if and only if

$$
\gamma=\frac{n}{\alpha}-\frac{n}{\beta}
$$

Remark 2. In fact, the condition $\gamma=\frac{n}{\alpha}-\frac{n}{\beta}$ is necessary for the boundedness of fractional integral operators $I_{\gamma}$. Let $\delta_{t} f(x)=f(t x)$, where $(t>0)$. Then,

$$
\begin{gathered}
I_{\gamma}\left(\delta_{t} f\right)=t^{-\gamma} \delta_{t} I_{\gamma}(f) \\
\left\|\delta_{t^{-1}} f\right\|_{\left(L^{\vec{q}}, L^{\vec{s}}\right)^{\beta}}=t^{\frac{n}{\beta}-\frac{1}{n} \sum_{i=1}^{n} \frac{1}{s_{i}}\|f\|_{\left(L^{\vec{q}}, L^{\vec{s}}\right)^{\beta}} .} \\
\left\|\delta_{t} f\right\|_{\left(L^{\vec{p}}, \vec{L}^{\vec{p}}\right)^{\alpha}}=t^{-\frac{n}{\alpha}+\sum_{i=1}^{n} \frac{1}{r_{i}}\|f\|_{\left(L^{\vec{p}}, L^{\vec{L}}\right)^{\alpha}} .}
\end{gathered}
$$

Thus, by the boundedness of $I_{\gamma}$ from $\left(L^{\vec{p}}, L^{\vec{r}}\right)^{\alpha}\left(\mathbb{R}^{n}\right)$ to $\left(L^{\vec{q}}, L^{\vec{s}}\right)^{\beta}\left(\mathbb{R}^{n}\right)$,

$$
\begin{aligned}
& \left\|I_{\gamma} f\right\|_{\left(L^{\vec{q}}, L^{\vec{s}}\right)^{\beta}}=t^{\gamma}\left\|\delta_{t^{-1}} I_{\gamma}\left(\delta_{t} f\right)\right\|_{\left(L^{\vec{q}}, L^{\vec{s}}\right)^{\beta}} \\
& =t^{\gamma+\frac{n}{\beta}-\sum_{i=1}^{n} \frac{1}{s_{i}}}\left\|I_{\gamma}\left(\delta_{t} f\right)\right\|_{\left(L^{\vec{q}}, L^{\vec{s}}\right)^{\beta}} \\
& \lesssim t^{\gamma+\frac{n}{\beta}-\sum_{i=1}^{n} \frac{1}{s_{i}}}\left\|\delta_{t} f\right\|_{\left(L^{\vec{p}}, L^{\vec{r}}\right)^{\alpha}}
\end{aligned}
$$

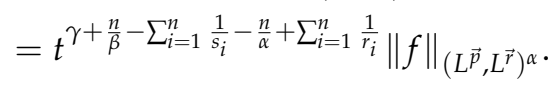

Thus, $\gamma=\frac{n}{\alpha}-\sum_{i=1}^{n} \frac{1}{r_{i}}-\frac{n}{\beta}+\sum_{i=1}^{n} \frac{1}{s_{i}}$ and $\gamma=\frac{n}{\alpha}-\frac{n}{\beta}$ when $\vec{s}=\vec{r}$.

Let $\left[b, I_{\gamma}\right]$ be the linear commutators generated by $I_{\gamma}$ and $B M O$ function $b$. We have the following result for the strong-type estimates of $\left[b, I_{\gamma}\right]$ on the mixed-norm amalgam spaces.

Theorem 4. Let $0<\gamma<n, 1<\vec{p} \leq \vec{q}<\infty, 1<\vec{s} \leq \infty, \frac{1}{n} \sum_{i=1}^{n} \frac{1}{s_{i}} \leq \frac{1}{\alpha} \leq \frac{1}{n} \sum_{i=1}^{n} \frac{1}{p_{i}}$, and $\frac{1}{n} \sum_{i=1}^{n} \frac{1}{s_{i}} \leq \frac{1}{\beta} \leq \frac{1}{n} \sum_{i=1}^{n} \frac{1}{q_{i}}$. Assume that $\gamma=\sum_{i=1}^{n} \frac{1}{p_{i}}-\sum_{i=1}^{n} \frac{1}{q_{i}}=\frac{n}{\alpha}-\frac{n}{\beta}$. If $b \in B M O\left(\mathbb{R}^{n}\right)$, then the linear commutators $\left[b, I_{\gamma}\right]$ are bounded from $\left(L^{\vec{p}}, L^{\vec{s}}\right)^{\alpha}\left(\mathbb{R}^{n}\right)$ to $\left(L^{\vec{q}}, L^{\vec{s}}\right)^{\beta}\left(\mathbb{R}^{n}\right)$.

In fact, if the linear commutators $\left[b, I_{\gamma}\right]$ are bounded from $\left(L^{\vec{p}}, L^{\vec{s}}\right)^{\alpha}\left(\mathbb{R}^{n}\right)$ to $\left(L^{\vec{q}}, L^{\vec{s}}\right)^{\beta}\left(\mathbb{R}^{n}\right)$, then $b \in B M O\left(\mathbb{R}^{n}\right)$. This result can be stated as follows.

Theorem 5. Let $0<\gamma<n, 1<\vec{p} \leq \vec{q}<\infty, 1<\vec{s} \leq \infty, \frac{1}{n} \sum_{i=1}^{n} \frac{1}{s_{i}} \leq \frac{1}{\alpha} \leq \frac{1}{n} \sum_{i=1}^{n} \frac{1}{p_{i}}$, and $\frac{1}{n} \sum_{i=1}^{n} \frac{1}{s_{i}} \leq \frac{1}{\beta} \leq \frac{1}{n} \sum_{i=1}^{n} \frac{1}{q_{i}}$. Assume that $\gamma=\sum_{i=1}^{n} \frac{1}{p_{i}}-\sum_{i=1}^{n} \frac{1}{q_{i}}=\frac{n}{\alpha}-\frac{n}{\beta}$. If the linear commutators $\left[b, I_{\gamma}\right]$ are bounded from $\left(L^{\vec{p}}, L^{\vec{s}}\right)^{\alpha}\left(\mathbb{R}^{n}\right)$ to $\left(L^{\vec{q}}, L^{\vec{s}}\right)^{\beta}\left(\mathbb{R}^{n}\right)$, then $b \in B M O\left(\mathbb{R}^{n}\right)$. 
Theorem 5 is proved by Proposition 5 and Theorem 1. By this new result, we can obtain the following result.

Corollary 1. Let $0<\gamma<n, 1<\vec{p} \leq \vec{q}<\infty, 1<\vec{s} \leq \infty, \frac{1}{n} \sum_{i=1}^{n} \frac{1}{s_{i}} \leq \frac{1}{\alpha} \leq \frac{1}{n} \sum_{i=1}^{n} \frac{1}{p_{i}}$, and $\frac{1}{n} \sum_{i=1}^{n} \frac{1}{s_{i}} \leq \frac{1}{\beta} \leq \frac{1}{n} \sum_{i=1}^{n} \frac{1}{q_{i}}$. If $\gamma=\sum_{i=1}^{n} \frac{1}{p_{i}}-\sum_{i=1}^{n} \frac{1}{q_{i}}=\frac{n}{\alpha}-\frac{n}{\beta}$, then the following statements are equivalent:

(i) The linear commutators $\left[b, I_{\gamma}\right]$ are bounded from $\left(L^{\vec{p}}, L^{\vec{s}}\right)^{\alpha}\left(\mathbb{R}^{n}\right)$ to $\left(L^{\vec{q}}, L^{\vec{s}}\right)^{\beta}\left(\mathbb{R}^{n}\right)$;

(ii) $b \in B M O\left(\mathbb{R}^{n}\right)$.

\section{Some Basic Properities}

In this section, we give proofs of the properties of mixed-norm amalgam spaces.

Proof of Proposition 1. First, we will check the triangle inequality. For $f, g \in\left(L^{\vec{p}}, L^{\vec{s}}\right)^{\alpha}\left(\mathbb{R}^{n}\right)$,

$$
\begin{aligned}
& \|f+g\|_{\left(L^{\vec{p}}, L^{\vec{s}}\right)^{\alpha}}=\sup _{r>0}\left\||B(\cdot, r)|^{\frac{1}{\alpha}-\frac{1}{n} \sum_{i=1}^{n} \frac{1}{p_{i}}-\frac{1}{n} \sum_{i=1}^{n} \frac{1}{s_{i}}}\right\|(f+g) \chi_{B(\cdot, r)}\left\|_{L^{\vec{p}}}\right\|_{L^{\vec{s}}} \\
& \leq \sup _{r>0}\left\||B(\cdot, r)|^{\frac{1}{\alpha}-\frac{1}{n} \sum_{i=1}^{n} \frac{1}{p_{i}}-\frac{1}{n} \sum_{i=1}^{n} \frac{1}{s_{i}}}\right\| f \chi_{B(\cdot, r)}\left\|_{L^{\vec{p}}}\right\|_{L^{\vec{s}}}
\end{aligned}
$$

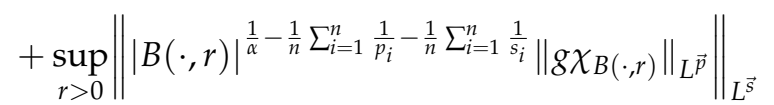

$$
\begin{aligned}
& =\|f\|_{\left(L^{\vec{p}}, L^{\vec{s}}\right)^{\alpha}}+\|g\|_{\left(L^{\vec{p}}, L^{\vec{s}}\right)^{\alpha}} .
\end{aligned}
$$

The positivity and the homogeneity are both clear. Thus, we prove that $\left(L^{\vec{p}}, L^{\vec{s}}\right)^{\alpha}\left(\mathbb{R}^{n}\right)$ are spaces with norm $\|\cdot\|_{\left(L^{\vec{p}}, L^{\vec{s}}\right)^{\alpha}}$. It remains to check the completeness. Without losing the generality, let a Cauchy sequence $\left\{f_{j}\right\}_{j=1}^{\infty} \subset\left(L^{\vec{p}}, L^{\vec{s}}\right)^{\alpha}\left(\mathbb{R}^{n}\right)$ satisfy

$$
\left\|f_{j+1}-f_{j}\right\|_{\left(L^{\vec{p}}, L^{\vec{s}}\right)^{\alpha}}<2^{-j} .
$$

We write $f=f_{1}+\sum_{j=1}^{\infty}\left(f_{j+1}-f_{j}\right)=\lim _{j \rightarrow \infty} f_{j}$. Then,

$$
\|f\|_{\left(L^{\vec{p}}, L^{\vec{s}}\right)^{\alpha}} \leq\left\|f_{1}\right\|_{\left(L^{\left.\vec{p}, L^{\vec{s}}\right)^{\alpha}}\right.}+\sum_{j=1}^{\infty}\left\|f_{j+1}-f_{j}\right\|_{\left(L^{\left.\vec{p}, L^{\vec{s}}\right)^{\alpha}}\right.}<\infty .
$$

Thus, for almost everywhere $x \in \mathbb{R}^{n}$,

$$
f(x)=f_{1}(x)+\sum_{j=1}^{\infty}\left(f_{j+1}(x)-f_{j}(x)\right) \leq\left|f_{1}(x)\right|+\sum_{j=1}^{\infty}\left|f_{j+1}(x)-f_{j}(x)\right|<\infty
$$

and $f \in\left(L^{\vec{p}}, L^{\vec{s}}\right)^{\alpha}\left(\mathbb{R}^{n}\right)$. Furthermore,

$$
\begin{aligned}
\left\|f-f_{J}\right\|_{\left(L^{\vec{p}}, L^{\vec{s}}\right)^{\alpha}} & =\left\|\sum_{j=1}^{\infty}\left(f_{j+1}-f_{j}\right)-\sum_{j=1}^{J-1}\left(f_{j+1}-f_{j}\right)\right\|_{\left(L^{\vec{p}}, L^{\vec{s}}\right)^{\alpha}} \\
& \leq \sum_{j=J}^{\infty}\left\|f_{j+1}-f_{j}\right\|_{\left(L^{\vec{p}}, L^{\vec{s}}\right)^{\alpha}} \\
& \leq 2 \cdot 2^{-J}
\end{aligned}
$$

and

$$
\lim _{J \rightarrow \infty}\left\|f-f_{J}\right\|_{\left(L^{\vec{p}}, L^{\vec{s}}\right)^{\alpha}}=0 .
$$

So, we prove that $\left(L^{\vec{p}}, L^{\vec{s}}\right)^{\alpha}$ are Banach spaces. By the same discussion, we can prove $\left(L^{\vec{p}}, L^{\vec{s}}\right)\left(\mathbb{R}^{n}\right)$ are also Banach spaces. 
Proof of Proposition 2. We prove these by contradiction. In fact, by the Lebesgue differential theorem in the mixed-norm Lebesgue spaces [16], we know

$$
\lim _{r \rightarrow 0} \frac{\left\|f \chi_{B(x, r)}\right\|_{L^{\vec{p}}}}{\left\|\chi_{B(x, r)}\right\|_{L^{\vec{p}}}}=f(x) \text { a.e. } x \in \mathbb{R}^{n} .
$$

Thus, if $\frac{1}{n} \sum_{i=1}^{n} \frac{1}{s_{i}}-\frac{1}{\alpha}>0$ and $f \neq 0$,

$$
\lim _{r \rightarrow 0}|B(y, r)|^{\frac{1}{\alpha}-\frac{1}{n} \sum_{i=1}^{n} \frac{1}{s_{i}}} \frac{\left\|f \chi_{B(x, r)}\right\|_{L^{\vec{p}}}}{\left\|\chi_{B(x, r)}\right\|_{L^{\vec{p}}}}=\infty .
$$

Therefore, we prove $\frac{1}{n} \sum_{i=1}^{n} \frac{1}{s_{i}}<\frac{1}{\alpha}$.

If $\frac{1}{\alpha}-\frac{1}{n} \sum_{i=1}^{n} \frac{1}{p_{i}}>0$, then we claim $\left\|\chi_{B\left(x_{0}, r_{0}\right)}\right\|_{\left(L^{\vec{p}}, L^{\vec{s}}\right)^{\alpha}}=\infty$ for any ball $B\left(x_{0}, r_{0}\right)$, which shows that $\left(L^{\vec{p}}, L^{\vec{s}}\right)^{\alpha}\left(\mathbb{R}^{n}\right)$ are trivial spaces. Hence, we acquire $\frac{1}{\alpha} \leq \frac{1}{n} \sum_{i=1}^{n} \frac{1}{p_{i}}$. Indeed, if $x \in B\left(x_{0}, \frac{r}{2}\right)$ and $2 r_{0}<r$, then for any $y \in B\left(x_{0}, r_{0}\right)$, we have

$$
|x-y| \leq\left|x_{0}-x\right|+\left|x_{0}-y\right| \leq \frac{r}{2}+r_{0}<r,
$$

that is $B\left(x_{0}, r_{0}\right) \subset B(x, r)$. Therefore,

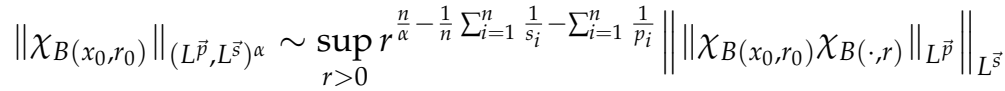

$$
\begin{aligned}
& \geq \sup _{r>2 r_{0}} r^{\frac{n}{\alpha}-\frac{1}{n} \sum_{i=1}^{n} \frac{1}{s_{i}}-\sum_{i=1}^{n} \frac{1}{p_{i}}}\left\|\chi_{B\left(x_{0}, \frac{r}{2}\right)}\right\| \chi_{B\left(x_{0}, r_{0}\right)}\left\|_{L^{\vec{p}}}\right\|_{L^{\vec{s}}} \\
& \gtrsim \sup _{r>2 r_{0}} r^{\frac{n}{\alpha}-\frac{1}{n} \sum_{i=1}^{n} \frac{1}{s_{i}}-\sum_{i=1}^{n} \frac{1}{p_{i}} \cdot r^{\frac{1}{n}} \sum_{i=1}^{n} \frac{1}{s_{i}}} \\
& \geq \lim _{r \rightarrow+\infty} r^{\frac{n}{\alpha}-\sum_{i=1}^{n} \frac{1}{p_{i}}} \\
& =+\infty \text {. }
\end{aligned}
$$

For the opposite side, by calculation, we can prove $\chi_{B(0,1)} \in\left(L^{\vec{p}}, L^{\vec{s}}\right)^{\alpha}\left(\mathbb{R}^{n}\right)$ if $\frac{1}{n} \sum_{i=1}^{n} \frac{1}{s_{i}} \leq$ $\frac{1}{\alpha} \leq \frac{1}{n} \sum_{i=1}^{n} \frac{1}{p_{i}}$.

Proof of Proposition 3. By direct calculation, we have

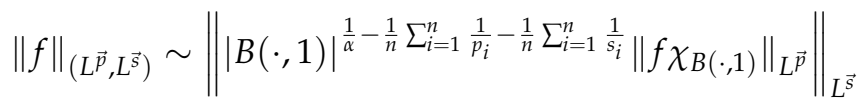

$$
\begin{aligned}
& \leq \sup _{r>0}\left\||B(\cdot, r)|^{\frac{1}{\alpha}-\frac{1}{n} \sum_{i=1}^{n} \frac{1}{p_{i}}-\frac{1}{n} \sum_{i=1}^{n} \frac{1}{s_{i}}}\right\| f \chi_{B(\cdot, r)}\left\|_{L^{\vec{p}}}\right\|_{L^{\vec{s}}} \\
& =\|f\|_{\left(L^{\vec{p}}, L^{\vec{s}}\right)^{\alpha}} .
\end{aligned}
$$

Therefore, $\left(L^{\vec{p}}, L^{\vec{s}}\right)^{\alpha}\left(\mathbb{R}^{n}\right) \subset\left(L^{\vec{p}}, L^{\vec{s}}\right)\left(\mathbb{R}^{n}\right)$ with $\|f\|_{\left(L^{\vec{p}}, L^{\vec{s}}\right)} \leq\|f\|_{\left(L^{\vec{p}}, L^{\vec{s}}\right)^{\alpha}}$. Particularly, if $\vec{p} \leq \vec{q}$, by Hölder's inequality,

$$
|B(x, r)|^{\frac{1}{\alpha}-\frac{1}{n} \sum_{i=1}^{n} \frac{1}{p_{i}}-\frac{1}{n} \sum_{i=1}^{n} \frac{1}{s_{i}}}\left\|f \chi_{B(x, r)}\right\|_{L^{\vec{p}}} \leq|B(x, r)|^{\frac{1}{\alpha}-\frac{1}{n} \sum_{i=1}^{n} \frac{1}{q_{i}}-\frac{1}{n} \sum_{i=1}^{n} \frac{1}{s_{i}}}\left\|f \chi_{B(x, r)}\right\|_{L^{\vec{q}}}
$$

Thus, $\|f\|_{\left(L^{\vec{p}}, L^{\vec{s}}\right)^{\alpha}} \leq\|f\|_{\left(L^{\vec{q}, L^{\vec{s}}}\right)^{\alpha}}$ and $\left(L^{\vec{q}}, L^{\vec{s}}\right)^{\alpha}\left(\mathbb{R}^{n}\right) \subseteq\left(L^{\vec{p}}, L^{\vec{s}}\right)^{\alpha}\left(\mathbb{R}^{n}\right)$.

Now, we show the proof of Proposition 4. 
Proof of Proposition 4. It is obvious that

$$
\left\|\chi_{B\left(x_{0}, r_{0}\right)}\right\|_{\left(L^{\vec{p}}, L^{\vec{s}}\right)^{\alpha}} \sim \sup _{r>0} r^{\frac{n}{\alpha}-\sum_{i=1}^{n} \frac{1}{s_{i}}-\sum_{i=1}^{n} \frac{1}{p_{i}}}\|\| \chi_{B\left(x_{0}, r_{0}\right)} \chi_{B(x, r)}\left\|_{L^{\vec{p}}}\right\|_{L^{\vec{s}}}
$$

If $r>r_{0}$, then by $\frac{1}{\alpha}-\frac{1}{n} \sum_{i=1}^{n} \frac{1}{p_{i}} \leq 0$

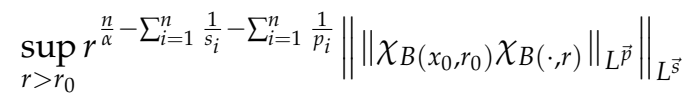

$$
\begin{aligned}
& \leq \sup _{r>r_{0}} r^{\frac{n}{\alpha}-\sum_{i=1}^{n} \frac{1}{s_{i}}-\sum_{i=1}^{n} \frac{1}{p_{i}}}\|\| \chi_{B\left(x_{0}, r_{0}\right)}\left\|_{L^{\vec{p}}} \cdot \chi_{B\left(x_{0}, r+r_{0}\right)}\right\|_{L^{\vec{s}}} \\
& \lesssim r_{0}^{\sum_{j=1}^{n} \frac{1}{p_{j}}} \sup _{r>r_{0}} r^{\frac{n}{\alpha}-\sum_{i=1}^{n} \frac{1}{s_{i}}-\sum_{i=1}^{n} \frac{1}{p_{i}}}\left(r+r_{0}\right)^{\sum_{i=1}^{n} \frac{n}{s_{i}}} \\
& =r_{0}^{\sum_{j=1}^{n} \frac{1}{p_{j}}} \sup _{r>r_{0}} r^{\frac{n}{\alpha}-\sum_{i=1}^{n} \frac{1}{p_{i}}}\left(1+\frac{r_{0}}{r}\right)^{\sum_{i=1}^{n} \frac{1}{s_{i}}} \\
& \lesssim r_{0}^{\frac{n}{\alpha}} \text {. }
\end{aligned}
$$

For $r \leq r_{0}$, by $\frac{1}{\alpha}-\frac{1}{n} \sum_{i=1}^{n} \frac{1}{s_{i}} \geq 0$, we have

$$
\begin{aligned}
& \sup _{r \leq r_{0}} r^{\frac{n}{\alpha}-\sum_{i=1}^{n} \frac{1}{s_{i}}-\sum_{i=1}^{n} \frac{1}{p_{i}}}\|\| \chi_{B\left(x_{0}, r_{0}\right)} \chi_{B(\cdot, r)}\left\|_{L^{\vec{p}}}\right\|_{L^{\vec{s}}}
\end{aligned}
$$

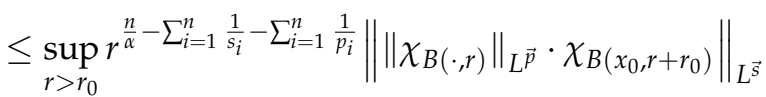

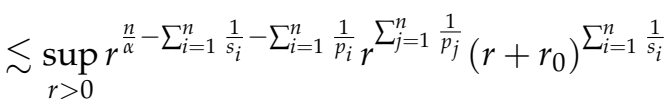

$$
\begin{aligned}
& =\sup _{r>0} r^{\frac{n}{\alpha}-\sum_{i=1}^{n} \frac{1}{p_{i}}}\left(r+r_{0}\right)^{\sum_{i=1}^{n} \frac{1}{s_{i}}} \\
& \lesssim r_{0}^{\frac{n}{\alpha}} \text {. }
\end{aligned}
$$

Thus, $\left\|\chi_{B\left(x_{0}, r_{0}\right)}\right\|_{\left(L^{\vec{p}}, L^{\vec{s}}\right)^{\alpha}} \lesssim r_{0}^{n / \alpha}$.

Next, we show that $\left\|\chi_{B\left(x_{0}, r_{0}\right)}\right\|_{\mathcal{H}\left(\vec{p}, \vec{s}^{\prime}, \alpha^{\prime}\right)} \lesssim r_{0}^{n / \alpha^{\prime}}$. First, by the similar argument dilation operator of (9), let

$$
\chi_{B\left(x_{0}, r_{0}\right)}=r^{\frac{n}{a^{\prime}}}\left\|\chi_{B\left(x_{0} / r, r_{0} / r\right)}\right\|_{\vec{p}^{\prime}, \vec{s}^{\prime}} \cdot S t_{r}^{\alpha^{\prime}}\left(\left\|\chi_{B\left(x_{0} / r, r_{0} / r\right)}\right\|_{\vec{p}^{\prime}, \vec{s}^{\prime}}^{-1} \chi_{B\left(x_{0} / r, r_{0} / r\right)}\right) .
$$

It is obvious that

$$
\|\| \chi_{B\left(x_{0} / r, r_{0} / r\right)}\left\|_{\vec{p}^{\prime}, \vec{s}^{\prime}}^{-1} \chi_{B\left(x_{0} / r, r_{0} / r\right)}\right\|_{\vec{p}^{\prime}, \vec{s}^{\prime}} \leq 1
$$

From Definition 2 and Proposition 5,

$$
\begin{aligned}
\left\|\chi_{B\left(x_{0}, r_{0}\right)}\right\|_{\mathcal{H}\left(\vec{p}^{\prime}, \vec{s}^{\prime}, \alpha^{\prime}\right)} & \leq \sup _{r>0} r \frac{n}{a^{\prime}}\left\|\chi_{B\left(x_{0} / r, r_{0} / r\right)}\right\|_{\vec{p}^{\prime}, \vec{s}^{\prime}} \\
& \lesssim \sup _{r>0} r \frac{n}{a^{\prime}}\|\| \chi_{B\left(x_{0} / r, r_{0} / r\right)} \chi_{B(\cdot, 1)}\left\|_{L^{\vec{p}^{\prime}}}\right\|_{L^{\vec{s}^{\prime}}} .
\end{aligned}
$$

Using the same argument of the proof of $\left\|\chi_{B\left(x_{0}, r_{0}\right)}\right\|_{\left(L^{\vec{p}}, L^{\vec{L}}\right)^{\alpha}} \lesssim r_{0}^{n / \alpha}$ with $r_{0} / r>1$ and $r_{0} / r \leq 1$, we have

$$
\left\|\chi_{B\left(x_{0}, r_{0}\right)}\right\|_{\mathcal{H}\left(\vec{p}^{\prime}, \vec{s}^{\prime}, \alpha^{\prime}\right)} \lesssim \sup _{r>0} r^{\frac{n}{\alpha^{\prime}}}\|\| \chi_{B\left(x_{0} / r, r_{0} / r\right)} \chi_{B(\cdot, 1)}\left\|_{L^{\vec{p}^{\prime}}}\right\|_{L^{\vec{s}^{\prime}}} \lesssim r_{0}^{n / \alpha^{\prime}}
$$


The proof is completed.

Before the proof of Proposition 5, the following two lemmas are necessary.

Lemma 3. Let $1 \leq \vec{p}, \vec{s} \leq \infty$ and $\frac{1}{n} \sum_{i=1}^{n} \frac{1}{s_{i}} \leq \frac{1}{\alpha} \leq \frac{1}{n} \sum_{i=1}^{n} \frac{1}{p_{i}}$. For any constant $\rho \in(0, \infty)$, we have

$$
\|\| f \chi_{B(\cdot, r)}\left\|_{L^{\vec{p}}}\right\|_{L^{\vec{s}}} \sim\|\| f \chi_{B(\cdot, \rho r)}\left\|_{L^{\vec{p}}}\right\|_{L^{\vec{s}}}
$$

where the positive equivalence constants are independent of $f$ and $t$.

Proof. Firstly, we prove the lemma holds when $\rho>1$. It is obvious that

$$
\|\| f \chi_{B(\cdot, r)}\left\|_{L^{\vec{p}}}\right\|_{L^{\vec{s}}} \leq\|\| f \chi_{B(\cdot, \rho r)}\left\|_{L^{\vec{p}}}\right\|_{L^{\vec{s}}} .
$$

Next, we prove the reverse inequality. We can find $N \in \mathbb{N}$ and $\left\{x_{1}, x_{2}, \cdots, x_{N}\right\}$, such that

$$
B(0, \rho r) \subset \bigcup_{j=1}^{N} B\left(x_{j}, r\right),
$$

where $N$ is independent of $r$ and $N \lesssim 1$. Therefore, we have

$$
\left\|f \chi_{B(x, \rho r)}\right\|_{L^{\vec{p}}} \leq\left\|f \sum_{j=1}^{N} \chi_{B\left(x+x_{j}, r\right)}\right\|_{L^{\vec{p}}} \leq \sum_{j=1}^{N}\left\|f \chi_{B\left(x+x_{j}, r\right)}\right\|_{L^{\vec{p}}}
$$

for any $x \in \mathbb{R}^{n}$. According to the translation invariance of the Lebesgue measure and $N \lesssim 1$, it follows that

$$
\|\| f \chi_{B(\cdot, \rho r)}\left\|_{L^{\vec{p}}}\right\|_{L^{\vec{s}}} \leq \sum_{j=1}^{N}\|\| f \chi_{B\left(\cdot+x_{j}, r\right)}\left\|_{L^{\vec{p}}}\right\|_{L^{\vec{s}}} \lesssim\|\| f \chi_{B(\cdot, r)}\left\|_{L^{\vec{p}}}\right\|_{L^{\vec{s}}} .
$$

For the $\rho \in(0,1)$, we only need replace $r$ by $r / \rho$. The proof is completed.

Remark 3. If taking $r=1$, we have

$$
\|f\|_{\left(L^{\vec{p}}, L^{\vec{S}}\right)} \sim\|\| f \chi_{B(\cdot, \rho)}\left\|_{L^{\vec{p}}}\right\|_{L_{L^{\vec{S}^{\prime}}}} \rho \in(0, \infty),
$$

where the positive equivalence constants are independent of $f$.

The following result plays an indispensable role in the proof of Proposition 5.

Lemma 4. Let $1 \leq \vec{p}, \vec{s} \leq \infty$ and $\frac{1}{n} \sum_{i=1}^{n} \frac{1}{s_{i}} \leq \frac{1}{\alpha} \leq \frac{1}{n} \sum_{i=1}^{n} \frac{1}{p_{i}}$. Then, we have

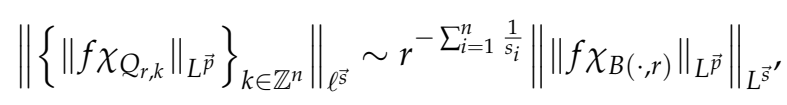

where the positive equivalence constants are independent of $f$ and $t$.

Proof. By Lemma 3, we only need to show that

$$
\left\|\left\{\left\|f \chi_{Q_{r, k}}\right\|_{L^{\vec{p}}}\right\}_{k \in \mathbb{Z}^{n}}\right\|_{\ell^{\vec{s}}} \sim r^{-\sum_{i=1}^{n} \frac{1}{s}}\|\| f \chi_{B(\cdot, 2 \sqrt{n} r)}\left\|_{L^{\vec{p}}}\right\|_{L^{\vec{s}}} .
$$

For any given $x \in \mathbb{R}^{n}$, we let

$$
A_{x}:=\left\{k \in \mathbb{Z}: Q_{r, k} \cap B(x, 2 \sqrt{n} r) \neq \varnothing\right\} .
$$


Then the cardinality of $A_{x}$ is finite and $x \in B(r k, 4 \sqrt{n} r)$ for any $k \in A_{x}$. Thus,

$$
\begin{aligned}
\left\|f \chi_{B(x, 2 \sqrt{n} r)}\right\|_{L^{\vec{p}}} & \leq\left\|\sum_{k \in A_{x}} f \chi_{Q_{r, k}}\right\|_{L^{\vec{p}}} \leq \sum_{k \in A_{x}}\left\|f \chi_{Q_{r, k}}\right\|_{L^{\vec{p}}} \\
& \leq \sum_{k \in \mathbb{Z}^{n}} \| f \chi_{Q_{r, k} \|_{L^{\vec{p}}} \chi_{B(r k, 4 \sqrt{n} r)}(x) .}
\end{aligned}
$$

Taking the $L^{\vec{s}}$-norm on $x$, we have

$$
\|\| f \chi_{B(\cdot, 2 \sqrt{n} r)}\left\|_{L^{\vec{p}}}\right\|_{L^{\vec{s}}} \leq\left\|\sum_{k \in \mathbb{Z}^{n}}\right\| f \chi_{Q_{r, k}}\left\|_{L^{\vec{p}}} \chi_{B(r k, 4 \sqrt{n} r)}\right\|_{L^{\vec{s}}} .
$$

By the similar argument of Lemma 3 , there exist $N \in \mathbb{N}$ and $\left\{k_{1}, k_{2}, \cdots, k_{N}\right\}$, such that

$$
B(0,4 \sqrt{n} r) \subset \bigcup_{j=1}^{N} Q_{k_{j}, r}
$$

where $N$ is independent of $r$ and $N \sim 1$. According to the translation invariance of the Lebesgue measure and $N \sim 1$, it follows that

$$
\begin{aligned}
& \|\| f \chi_{B(\cdot, 2 \sqrt{n} r)}\left\|_{L^{\vec{p}}}\right\|_{L^{\vec{s}}} \leq\left\|\sum_{k \in \mathbb{Z}^{n}}\right\| f \chi_{Q_{r, k}}\left\|_{L^{\vec{p}}} \chi_{B(\rho k, 4 \sqrt{n} r)}\right\|_{L^{\vec{s}}} \\
& \leq\left\|\sum_{j=1}^{N} \sum_{k \in \mathbb{Z}^{n}}\right\| f \chi_{Q_{r, k}}\left\|_{L^{\vec{p}}} \chi_{Q_{r, k_{j}+k}}\right\|_{L^{\vec{s}}} \\
& \lesssim r^{\sum_{i=1}^{n} \frac{1}{s_{i}}}\left\|\left\{\left\|f \chi_{Q_{r, k}}\right\|_{L^{\vec{p}}}\right\}_{k \in \mathbb{Z}^{n}}\right\|_{\ell^{\vec{s}}} .
\end{aligned}
$$

Indeed, the last inequality is obtained by the following fact that

$$
\begin{aligned}
& \left(\int_{\mathbb{R}} \cdots\left(\int_{\mathbb{R}}\left|\sum_{k \in \mathbb{Z}^{n}} C_{k} \chi_{k r+(0, r]^{n}}(x)\right|^{s_{1}} d x_{1}\right)^{\frac{s_{2}}{s_{1}}} \cdots d x_{n}\right)^{\frac{1}{s_{n}}} \\
= & \left(\int_{\mathbb{R}} \cdots\left(\int_{\mathbb{R}}\left|\sum_{k \in \mathbb{Z}^{n}} C_{k} \prod_{i=1}^{n} \chi_{I_{k_{i}}}\left(x_{i}\right)\right|^{s_{1}} d x_{1}\right)^{\frac{1}{s_{1}}} \cdots d x_{n}\right)^{\frac{s_{2}}{s_{n}}} \\
= & \left(\sum_{k_{n} \in \mathbb{Z}^{\frac{1}{s_{n}}}} \int_{I_{k_{n}}} \ldots\left(\sum_{k_{1} \in \mathbb{Z}^{n}} \int_{I_{k_{1}}}\left|C_{k}\right|^{s_{1}} d x_{1}\right)^{\frac{s_{2}}{s_{1}}} \cdots d x_{n}\right)^{\frac{1}{s_{n}}}, \\
= & r^{\sum_{i=1}^{n} s_{i}} \cdot\left(\sum_{k_{n} \in \mathbb{Z}} \cdots\left(\sum_{k_{1} \in \mathbb{Z}^{n}}\left|C_{k}\right|^{s_{1}} d x_{1}\right)^{\frac{s_{2}}{s_{1}}} \cdots d x_{n}\right)^{,}
\end{aligned}
$$

where $C_{k}=\left\|f \chi_{Q_{r, k}}\right\|_{L^{\vec{p}}}$ and $I_{k_{i}}=r k_{i}+(0, r]$. Thus, we prove that

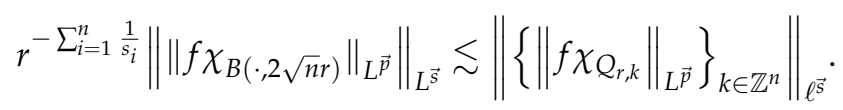


For the opposite inequality, it is obvious that

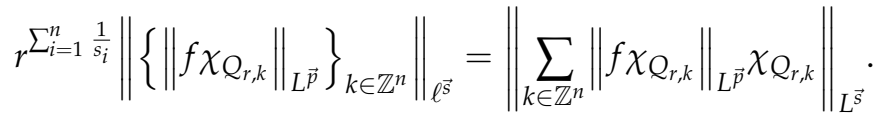

By $Q_{r, k} \subset B(x, 2 \sqrt{n} r)$ for $x \in Q_{r, k}$, we have

$$
r^{\sum_{i=1}^{n} \frac{1}{s_{i}}}\left\|\left\{\left\|f \chi_{Q_{r, k}}\right\|_{L^{\vec{p}}}\right\}_{k \in \mathbb{Z}^{n}}\right\|_{\ell^{\vec{s}}} \leq\|\| f \chi_{B(\cdot, 2 \sqrt{n} r)}\left\|_{L_{\vec{p}}}\right\|_{L^{\vec{s}}} .
$$

The proof is completed.

Lemma 4 can prove the proof of Proposition 5.

Proof of Proposition 5. According to Lemma 4, we obtain that

$$
\left\|\left\{\left\|f \chi_{Q_{1, k}}\right\|_{L^{\vec{p}}}\right\}_{k \in \mathbb{Z}^{n}}\right\|_{\ell^{\vec{s}}} \sim\|\| f \chi_{B(\cdot, 1)}\left\|_{L^{\vec{p}}}\right\|_{L^{\vec{s}}}
$$

and

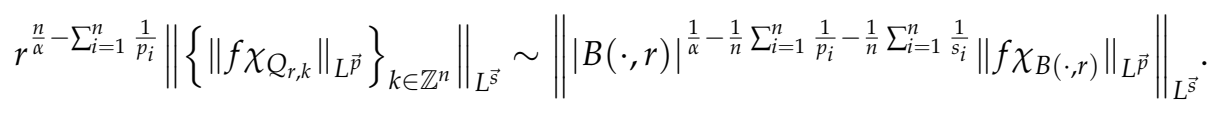

Thus, we prove Proposition 5.

\section{The Predual of Amalgam Spaces}

In this section, we will prove Theorem 1, whose ideal comes from [33]. Before that, the dual of mixed-norm amalgam spaces $\left(L^{\vec{p}}, L^{\vec{s}}\right)\left(\mathbb{R}^{n}\right)$ is given as follows.

Lemma 5. (i) Let $1 \leq \vec{p}, \vec{s} \leq \infty$. For $r \in(0, \infty)$, we have

$$
\|f g\|_{1} \leq_{r}\|f\|_{\vec{p}, \vec{s}} \cdot r\|g\|_{\vec{p}^{\prime}, \vec{s}^{\prime},} f, g \in L_{l o c}^{1}\left(\mathbb{R}^{n}\right)
$$

(ii) Let $1 \leq \vec{p}, \vec{s}<\infty$. The dual of mixed-norm amalgam spaces $\left(L^{\vec{p}}, \ell^{\vec{s}}\right)\left(\mathbb{R}^{n}\right)$ is $\left(L^{\vec{p}^{\prime}}, \ell^{\vec{s}^{\prime}}\right)\left(\mathbb{R}^{n}\right)$.

Proof. For $0<r<\infty$, by Hölder's inequality, we have

$$
\|f g\|_{1} \leq_{r}\|f\|_{\vec{p}, \vec{s}} \cdot r\|g\|_{\vec{p}^{\prime}, \vec{s}^{\prime}}, f, g \in L_{l o c}^{1}\left(\mathbb{R}^{n}\right) .
$$

According to ([19] Theorem 2) and ([1] Theorem 1a of Section 3), we deduce that the dual of $\left(L^{\vec{p}}, \ell^{s_{1}}\right)\left(\mathbb{R}^{n}\right)$ is $\left(L^{\vec{p}^{\prime}}, \ell^{s_{1}^{\prime}}\right)\left(\mathbb{R}^{n}\right)$. If the dual of $\left(L^{\vec{p}}, \ell^{\vec{s}}\right)\left(\mathbb{R}^{n}\right)$ is $\left(L^{\vec{p}^{\prime}}, \ell^{\bar{s}^{\prime}}\right)\left(\mathbb{R}^{n}\right)$ with $\bar{s}=\left(s_{1}, s_{2}, \cdots, s_{n-1}\right)$, using $([19]$ Theorem 2$)$,

$$
\begin{aligned}
\left(L^{\vec{p}}, \ell^{\vec{s}}\right)^{*} & =\left(\prod_{k_{n} \in \mathbb{Z}}\left(L^{\vec{p}}, \ell^{\bar{s}}\right), \ell^{s_{n}}\right)^{*}=\left(\prod_{k_{n} \in \mathbb{Z}}\left(L^{\vec{p}}, \ell^{\bar{s}}\right)^{*},\left(\ell^{s_{n}}\right)^{*}\right) \\
& =\left(\prod_{k_{n} \in \mathbb{Z}}\left(L^{\vec{p}^{\prime}}, \ell^{\bar{s}^{\prime}}\right), \ell^{s_{n}^{\prime}}\right)=\left(L^{\vec{p}^{\prime}}, \ell^{\vec{s}^{\prime}}\right) .
\end{aligned}
$$

Hence, $\left(L^{\vec{p}^{\prime}}, \ell^{\vec{s}^{\prime}}\right)\left(\mathbb{R}^{n}\right)$ is isometrically isomorphic to the dual of $\left(L^{\vec{p}}, \ell^{\vec{s}}\right)\left(\mathbb{R}^{n}\right)$. There is a unique element $\phi(T)$ of $\left(L^{\vec{p}}, \ell^{\vec{s}}\right)\left(\mathbb{R}^{n}\right)$ such that

$$
T(f)=\int_{\mathbb{R}^{n}} f(x) \phi(T)(x) d x, f \in\left(L^{\vec{p}}, \ell^{\vec{s}}\right)\left(\mathbb{R}^{n}\right)
$$

and, furthermore,

$$
\|\phi(T)\|_{\vec{p}^{\prime}, \vec{s}^{\prime}}=\|T\|
$$


where $\|T\|:=\sup \left\{|T(f)|: f \in L_{l o c}^{1}\left(\mathbb{R}^{n}\right)\right.$ and $\left.\|f\|_{\vec{p}, \vec{s}} \leq 1\right\}$.

Now, we discuss the properties of the dilation operator $S t_{r}^{(\alpha)}: f(x) \mapsto r^{-\frac{n}{\alpha}} f\left(r^{-1} x\right)$ for $0<\alpha<\infty$ and $0<r<\infty$. By direct computation, we have the following properties.

Proposition 6. Let $f \in L_{l o c}^{1}\left(\mathbb{R}^{n}\right), 0<\alpha<\infty$, and $0<r<\infty$.

(i) $S t_{r}^{(\alpha)}$ maps $L_{\text {loc }}^{1}\left(\mathbb{R}^{n}\right)$ into itself.

(ii) $f=S t_{1}^{(\alpha)}(f)$.

(iii) $S t_{r_{1}}^{(\alpha)} \circ S t_{r_{2}}^{(\alpha)}=S t_{r_{2}}^{(\alpha)} S t_{r_{2}}^{(\alpha)}=S t_{r_{1} r_{2}}^{(\alpha)}$.

(iv) $\sup _{r>0}\left\|S t_{r}^{(\alpha)}(f)\right\|_{\vec{p}, \vec{s}}=\|f\|_{\vec{p}, \vec{s}, \alpha}$, where $1 \leq \vec{p}, \vec{s}<\infty$ and $\frac{1}{n} \sum_{i=1}^{n} \frac{1}{s_{i}} \leq \frac{1}{\alpha} \leq \frac{1}{n} \sum_{i=1}^{n} \frac{1}{p_{i}}$.

Proposition 6 and Definition 2 prove the following result.

Proposition 7. Let $1 \leq \vec{p}, \vec{s} \leq \infty$, and $\frac{1}{n} \sum_{i=1}^{n} \frac{1}{s_{i}} \leq \frac{1}{\alpha} \leq \frac{1}{n} \sum_{i=1}^{n} \frac{1}{p_{i}} .\left(L^{\vec{p}^{\prime}}, \ell^{\vec{s}^{\prime}}\right)\left(\mathbb{R}^{n}\right)$ is a dense subspace of $\mathcal{H}\left(\vec{p}^{\prime}, \vec{s}^{\prime}, \alpha^{\prime}\right)$.

Proof. First, we verify that $\left(L^{\vec{p}^{\prime}}, \ell^{\vec{s}^{\prime}}\right)\left(\mathbb{R}^{n}\right)$ is continuously embedded into $\mathcal{H}\left(\vec{p}^{\prime}, s^{\prime}, \alpha^{\prime}\right)$. For any $f \in\left(L^{\vec{p}^{\prime}}, \ell^{\vec{s}^{\prime}}\right)\left(\mathbb{R}^{n}\right)$, we have

$$
f=\|f\|_{\vec{p}^{\prime}, \vec{s}^{\prime}} S t_{1}^{\alpha}\left(\|f\|_{\vec{p}^{\prime}, \vec{s}^{\prime}}^{-1} f\right)
$$

and

$$
\|\| f\left\|_{\vec{p}^{\prime}, \vec{s}^{\prime}}^{-1} f\right\|_{\vec{p}^{\prime}, \vec{s}^{\prime}}=1 \text {. }
$$

Thus, $f \in \mathcal{H}\left(\vec{p}^{\prime}, \vec{s}^{\prime}, \alpha^{\prime}\right)$ and satisfies

$$
\|f\|_{\mathcal{H}\left(\vec{p}^{\prime}, \vec{s}^{\prime}, \alpha^{\prime}\right)} \leq\|f\|_{\vec{p}^{\prime}, \vec{s}^{\prime}} .
$$

Let us show the denseness of $\left(L^{\vec{p}^{\prime}}, \ell^{\hat{s}^{\prime}}\right)\left(\mathbb{R}^{n}\right)$ in $\mathcal{H}\left(\vec{p}^{\prime}, \vec{s}^{\prime}, \alpha^{\prime}\right)$. It is clear that if $\left\{\left(c_{j}, r_{j}, f_{j}\right)\right\}_{j \geq 1}$ is a block decomposition of $f \in \mathcal{H}\left(\vec{p}^{\prime}, \vec{s}^{\prime}, \alpha^{\prime}\right)$, then

$$
\left\{\sum_{j=1}^{J} c_{j} S t_{r_{j}}^{\left(\alpha^{\prime}\right)}\left(f_{j}\right)\right\}_{J \geq 1} \subset\left(L^{\vec{p}^{\prime}}, \ell^{\vec{s}^{\prime}}\right)\left(\mathbb{R}^{n}\right)
$$

and

$$
\left\|f-\sum_{j=1}^{J} c_{j} S t_{r_{j}}^{\left(\alpha^{\prime}\right)}\left(f_{j}\right)\right\|_{\mathcal{H}\left(\vec{p}^{\prime}, \vec{s}^{\prime}, \alpha^{\prime}\right)}=\left\|\sum_{j=J+1}^{\infty} c_{j} S t_{r_{j}}^{\left(\alpha^{\prime}\right)}\left(f_{j}\right)\right\|_{\mathcal{H}\left(\vec{p}^{\prime}, \vec{s}^{\prime}, \alpha^{\prime}\right)} \leq \sum_{j=J+1}^{\infty}\left|c_{j}\right| \rightarrow 0
$$

with $J \rightarrow \infty$. Thus, $\left(L^{\vec{p}^{\prime}}, \ell^{\vec{s}^{\prime}}\right)\left(\mathbb{R}^{n}\right)$ is a dense subspace of $\mathcal{H}\left(\vec{p}^{\prime}, \vec{s}^{\prime}, \alpha^{\prime}\right)$.

Now, let us prove the main theorem in this section. 
Proof of Theorem 1. Let us prove (i). Let $\left\{\left(c_{j}, r_{j}, f_{j}\right)\right\}_{j \geq 1}$ be a block decomposition of $f$. By Proposition 5 and (7), we have for any $j \geq 1$

$$
\begin{aligned}
\left|\int_{\mathbb{R}^{n}} S t_{r_{j}}^{\left(\alpha^{\prime}\right)}\left(f_{j}\right)(x) g(x) d x\right| & =\left|\int_{\mathbb{R}^{n}} S t_{r_{j}^{-1}}^{(\alpha)}(g)(x) f_{j}(x) d x\right| \\
& \leq \int_{\mathbb{R}^{n}}\left|S t_{r_{j}^{-1}}^{(\alpha)}(g)(x) f_{j}(x)\right| d x \\
& \leq\left\|f_{j}\right\|_{\vec{p}^{\prime}, \vec{s}^{\prime}}\left\|S t_{r_{j}^{-1}}^{(\alpha)}(g)\right\|_{\vec{p}, \vec{s}} \\
& \leq\left\|S t_{r_{j}^{-1}}^{(\alpha)}(g)\right\|_{\vec{p}, \vec{s}} \leq\|g\|_{\vec{p}, \vec{s}, \alpha} .
\end{aligned}
$$

Therefore, we have

$$
\sum_{j \geq 1} \int_{\mathbb{R}^{n}}\left|c_{j} S t_{r_{j}}^{\left(\alpha^{\prime}\right)}\left(f_{j}\right)(x) g(x)\right| d x \leq\|g\|_{\vec{p}, \vec{s}, \alpha} \sum_{j \geq 1}\left|c_{j}\right| .
$$

Thus, we have $f g \in L^{1}\left(\mathbb{R}^{n}\right)$ and

$$
\left|\int_{\mathbb{R}^{n}} f(x) g(x) d x\right| \leq \int_{\mathbb{R}^{n}}|f(x) g(x)| d x \leq\|g\|_{\vec{p}, \vec{s}, \alpha} \sum_{j \geq 1}\left|c_{j}\right| .
$$

Taking the infimum with respect to all block decompositions of $f$, we obtain

$$
\left|\int_{\mathbb{R}^{n}} f(x) g(x) d x\right| \leq \int_{\mathbb{R}^{n}}|f(x) g(x)| d x \leq\|g\|_{\vec{p}, \vec{s}, \alpha}\|f\|_{\vec{p}^{\prime}, \vec{s}^{\prime}, \alpha^{\prime}} .
$$

Now, let us prove (ii). By (i), we have

$$
T_{g} \in \mathcal{H}(\vec{p}, \vec{s}, \alpha)^{*} .
$$

For any $a_{1}, a_{2} \in R, g_{1}, g_{2} \in\left(L^{\vec{p}}, \ell^{\vec{s}}\right)^{\alpha}\left(\mathbb{R}^{n}\right)$

$$
T\left(a_{1} g_{1}+a_{2} g_{2}\right)=a_{1} T_{g_{1}}+a_{2} T_{g_{2}}
$$

and

$$
\left\|T_{g}\right\|=\sup _{\|f\|_{\mathcal{H}(\vec{p}, \vec{s}, \alpha)} \leq 1}\left|T_{g}(f)\right| \leq\|g\|_{\vec{p}, \vec{s}, \alpha},
$$

that is, $T$ is linear and bounded mapping from $\left(L^{\vec{p}}, \ell^{\vec{s}}\right)^{\alpha}\left(\mathbb{R}^{n}\right)$ into $\mathcal{H}\left(\vec{p}^{\prime}, \vec{s}^{\prime}, \alpha^{\prime}\right)^{*}$ satisfying $\|T\| \leq 1$. For any $g_{1}, g_{2} \in\left(L^{\vec{p}}, \ell^{\vec{s}}\right)^{\alpha}\left(\mathbb{R}^{n}\right) \subset\left(L^{\vec{p}}, \ell^{\vec{s}}\right)\left(\mathbb{R}^{n}\right)$, if $T_{g_{1}}=T_{g_{2}}$, then for any $f \in$ $\left(L^{\vec{p}^{\prime}}, \ell^{\vec{s}^{\prime}}\right)\left(\mathbb{R}^{n}\right) \subset \mathcal{H}\left(\vec{p}^{\prime}, \vec{s}^{\prime}, \alpha^{\prime}\right)$, we have

$$
T_{g_{1}}(f)=T_{g_{2}}(f) .
$$

Thus, $g_{1}=g_{2}$, that is , $T$ is injective.

Now, we will prove that $T$ is a surjection and $\|g\|_{\vec{p}, \vec{s}, \alpha} \leq\left\|T_{g}\right\|$ (or $\|T\| \geq 1$ ). Let $T^{*}$ be an element of $\mathcal{H}\left(\vec{p}^{\prime}, \vec{s}^{\prime}, \alpha^{\prime}\right)^{*}$. From Proposition 7 , it follows that the restriction $T_{0}^{*}$ of $T^{*}$ to $\left(L^{\vec{p}^{\prime}}, \ell^{\vec{s}^{\prime}}\right)\left(\mathbb{R}^{n}\right)$ belongs to $\mathcal{H}\left(\vec{p}^{\prime}, \vec{s}^{\prime}, \alpha^{\prime}\right)^{*}$. Furthermore, we have

$$
\frac{1}{n} \sum_{j=1}^{n} \frac{1}{p_{i}^{\prime}} \leq \frac{1}{\alpha^{\prime}} \leq \frac{1}{n} \sum_{i=1}^{n} \frac{1}{s_{i}^{\prime}} .
$$


There is an element $g$ of $\left(L^{\vec{p}}, \ell^{\vec{s}}\right)\left(\mathbb{R}^{n}\right)$ such that for any $f \in\left(L^{\vec{p}^{\prime}}, \ell^{\vec{s}^{\prime}}\right)\left(\mathbb{R}^{n}\right)$

$$
T^{*}(f)=T_{0}^{*}(f)=\int_{\mathbb{R}^{n}} f(x) g(x) d x
$$

Hence, for $f \in\left(L^{\vec{p}^{\prime}}, \ell^{\vec{s}^{\prime}}\right)\left(\mathbb{R}^{n}\right)$ and $\rho>0$, we have

$$
\int_{\mathbb{R}^{n}} S t_{r}^{(\alpha)}(g)(x) f(x) d x=\int_{\mathbb{R}^{n}} g(x) S t_{r^{-1}}^{\left(\alpha^{\prime}\right)}(f)(x) d x=T^{*}\left[S t_{r^{-1}}^{\left(\alpha^{\prime}\right)}(f)\right] .
$$

and $S t_{r^{-1}}^{\left(\alpha^{\prime}\right)}(f) \in \mathcal{H}\left(\vec{p}^{\prime}, \vec{s}^{\prime}, \alpha^{\prime}\right)$. By the the assumption $T^{*} \in \mathcal{H}\left(\vec{p}^{\prime}, \vec{s}^{\prime}, \alpha^{\prime}\right)^{*}$, we have

$$
\left|\int_{\mathbb{R}^{n}} S t_{r}^{(\alpha)}(g)(x) f(x) d x\right| \leq\left\|T^{*}\right\| \cdot\left\|S t_{\rho^{-1}}^{\left(\alpha^{\prime}\right)}(f)\right\|_{\mathcal{H}\left(\vec{p}^{\prime}, \vec{s}^{\prime}, \alpha^{\prime}\right)} \leq\left\|T^{*}\right\| \cdot\|f\|_{\vec{p}^{\prime}, \vec{s}^{\prime}} .
$$

Due to (6), it follows that

$$
\left\|S t_{r}^{(\alpha)}(g)\right\|_{\vec{p}, \vec{s}} \leq\left\|T^{*}\right\| .
$$

Therefore, for any $g \in\left(L^{\vec{p}}, \ell^{\vec{s}}\right)\left(\mathbb{R}^{n}\right)$, by Proposition 6,

$$
\|g\|_{\vec{p}, \vec{s}, \alpha} \leq\left\|T^{*}\right\| \text {. }
$$

According to (10) and Proposition 7, we obtain

$$
T^{*}(f)=\int_{\mathbb{R}^{n}} f(x) g(x) d x, f \in \mathcal{H}\left(\vec{p}^{\prime}, \vec{s}^{\prime}, \alpha^{\prime}\right) .
$$

Thus, $T$ is a surjection and $\|g\|_{\vec{p}, \vec{s}, \alpha} \leq\|T\|$.

For (iii), the Hahn-Banach theorem shows that (6) holds.

\section{The Boundedness of Maximal Function}

In this section, we prove the boundedness of maximal function and discuss the rationality of fractional integral operators and their commutators on mixed-norm amalgam spaces.

Proof of Theorem 2. From Theorem 1.2 [10], we obtain that

$$
\|M f\|_{L^{\vec{p}}} \leq\left. C\|f\|\right|_{L_{\vec{p}}}
$$

Thus, let $B=B(y, r)$, for $f=f \chi_{3 B}+f \chi_{(3 B)^{c}}=: f_{1}+f_{2}$,

$$
\begin{aligned}
& |B(y, r)|^{\frac{1}{\beta}-\frac{1}{n} \sum_{i=1}^{n} \frac{1}{s_{i}}-\frac{1}{n} \sum_{i=1}^{n} \frac{1}{p_{i}}}\left\|M f \chi_{B(y, r)}\right\|_{L^{\vec{p}}}
\end{aligned}
$$

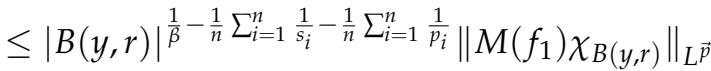

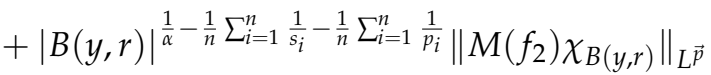

$$
\begin{aligned}
& :=\mathrm{I}(y, r)+\mathrm{II}(y, r) \text {. }
\end{aligned}
$$

For $\mathrm{I}(y, r)$, we have

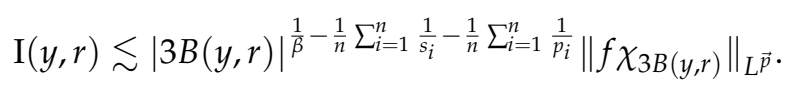

For II $(y, r)$, from

$$
M f_{2}(y) \lesssim \sup _{B \subseteq R} \frac{1}{|R|} \int_{R}|f(x)| d x, y \in B,
$$


we have

$$
\mathrm{II}(y, r)<\sup _{B \subseteq R} \frac{1}{|R|} \int_{R}|f(x)| d x \cdot|B(y, r)|^{\frac{1}{\alpha}-\frac{1}{n} \sum_{i=1}^{n} \frac{1}{s_{i}}},
$$

where $R$ denotes the open ball. According to the definition of sup, there exists $z \in B$ and $r_{1}>2 r$, such that

$$
\sup _{B \subseteq R} \frac{1}{|R|} \int_{R}|f(x)| d x<\frac{2}{\left|B\left(z, r_{1}\right)\right|} \int_{B\left(z, r_{1}\right)}|f(x)| d x .
$$

By $B\left(z, r_{1}\right) \subset B\left(y, 2 r_{1}\right)$,

$$
\sup _{B \subseteq R} \frac{1}{|R|} \int_{R}|f(x)| d x \lesssim \frac{1}{\left|B\left(y, 2 r_{1}\right)\right|} \int_{B\left(y, 2 r_{1}\right)}|f(x)| d x .
$$

Thus,

$$
\mathrm{II}(y, r) \lesssim\left|B\left(y, 2 r_{1}\right)\right|^{\frac{1}{\alpha}-1-\frac{1}{n} \sum_{i=1}^{n} \frac{1}{s_{i}}} \int_{B\left(y, 2 r_{1}\right)}|f(x)| d x .
$$

According to (11), (12), and Proposition 3(ii), we have

$$
\begin{aligned}
\|M f\|_{\left(L^{\vec{p}}, L^{\vec{L}}\right)^{\alpha}} & \leq \sup _{r>0}\|\mathrm{I}(\cdot, r)\|_{L^{\vec{s}}}+\sup _{r>0}\|\mathrm{II}(\cdot, r)\|_{L^{\vec{s}}} \\
& \lesssim\|f\|_{\left(L^{\vec{p}}, L^{\vec{s}}\right)^{\alpha}}+\|f\|_{\left(L^{1}, L^{\vec{s}}\right)^{\alpha}} \\
& \lesssim\|f\|_{\left(L^{\vec{p}}, L^{\vec{s}}\right)^{\alpha}} .
\end{aligned}
$$

The proof is complete.

To discuss the rationality of fractional integral operators and their commutators on mixed-norm amalgam spaces, we need the following lemmas about the $B M O\left(\mathbb{R}^{n}\right)$ function.

Lemma 6. Let $b$ be a function in $B M O\left(\mathbb{R}^{n}\right)$.

(i) For any ball $B$ in $\mathbb{R}^{n}$ and for any positive integer $j \in \mathbb{Z}^{+}$,

$$
\left|b_{2^{j+1} B}-b_{B}\right| \leq C j\|b\|_{B M O} .
$$

(ii) Let $1<\vec{p}<\infty$. There exist positive constants $C_{1} \leq C_{2}$ such that for all $b \in B M O\left(\mathbb{R}^{n}\right)$,

$$
C_{1}\|b\|_{B M O} \leq \sup _{B \subset \mathbb{R}^{n}} \frac{\left\|b-b_{B}\right\|_{L^{\vec{p}}\left(\mathbb{R}^{n}\right)}}{\left\|\chi_{B}\right\|_{L^{\vec{p}}\left(\mathbb{R}^{n}\right)}} \leq C_{2}\|b\|_{B M O}
$$

Proof. For (i), we have

$$
\begin{aligned}
\left|b_{2^{j+1} B}-b_{B}\right| & \leq \sum_{i=1}^{j}\left|b_{2^{i+1} B}-b_{2^{i} B}\right| \\
& \leq C \sum_{i=1}^{j} \frac{1}{\left|2^{i+1} B\right|} \int_{2^{i+1} B}\left|b(x)-b_{2^{i+1} B}\right| d x \\
& \leq 2 C j\|b\|_{B M O} .
\end{aligned}
$$

By Lemma 3.5 [34], the $M f$ is bounded on $L^{\vec{p}}\left(\mathbb{R}^{n}\right)$ with $1<\vec{p}=\left(p_{1}, p_{2}, \cdots, p_{n}\right)<\infty$. According to the dual theorem of Theorem 1.a [1], the associate space of $L^{\vec{p}}\left(\mathbb{R}^{n}\right)$ is $L^{\vec{p}^{\prime}}\left(\mathbb{R}^{n}\right)$. Finally, by Theorem 1.1 [35], the proof of (ii) can be proved.

Let $M_{t} f(x)=M\left(|f|^{t}\right)^{\frac{1}{t}}$. We only discuss the rationality of fractional integral operators' commutators on mixed-norm amalgam spaces. 
Let $f \in\left(L^{\vec{p}}, L^{\vec{s}}\right)^{\alpha}\left(\mathbb{R}^{n}\right), b \in B M O\left(\mathbb{R}^{n}\right)$. By the definition of $\left(L^{\vec{p}}, L^{\vec{s}}\right)^{\alpha}\left(\mathbb{R}^{n}\right)$, we have

$$
\|\| f \chi_{B(y, r)}\left\|_{L^{\vec{p}}}\right\|_{L^{\vec{s}}}<\infty .
$$

So there exists $B=B(y, r) \ni x$ such that $f \chi_{B(y, r)} \in L^{\vec{p}}\left(\mathbb{R}^{n}\right)(1<\vec{p}<\infty)$. For $\left[b, I_{\gamma}\right] f_{1}(x)=$ $b(x) I_{\gamma}\left(f \chi_{B}\right)(x)-I_{\gamma}\left(f \chi_{B}\right)(x)$, we have

$$
\left|I_{\gamma}\left(f \chi_{B}\right)(x)\right| \leq \int_{\mathbb{R}^{n}} \frac{\left|f(z) \chi_{B}(z)\right|}{|x-z|^{n-\gamma}} d z \leq C_{n} r^{\gamma} M f(x)
$$

and

$$
\begin{aligned}
\left|I_{\gamma}\left(f \chi_{B}\right)(x)\right| & \leq \int_{\mathbb{R}^{n}} \frac{\left|b(z) f(z) \chi_{B}(z)\right|}{|x-z|^{n-\gamma}} d z \\
& \leq b_{B} \int_{\mathbb{R}^{n}} \frac{\left|f(z) \chi_{B}(z)\right|}{|x-z|^{n-\gamma}} d z+\int_{\mathbb{R}^{n}} \frac{\left|b(z)-b_{B}\right|\left|f(z) \chi_{B}(z)\right|}{|x-z|^{n-\gamma}} d z \\
& \leq C_{n} b_{B} r^{\gamma} M f(x)+\left\|\left|b(\cdot)-b_{B}\right| \chi_{B}\right\|_{L^{t^{\prime}}}\left(\int_{\mathbb{R}^{n}} \frac{|f(z)|^{t} \chi_{B}(z)}{|x-z|^{t(n-\gamma)}} d z\right)^{\frac{1}{t}} \\
& \leq C_{n} b_{B} r^{\gamma} M f(x)+C_{n} r^{n-t(n-\gamma)+\frac{n}{t^{\prime}}\|b\|_{B M O} M f(x),}
\end{aligned}
$$

where $1<t<\frac{n}{n-\gamma}$ is small enough.

For $\left[b, I_{\gamma}\right]\left(f \chi_{B(y, r) c}\right)$,

$$
\begin{aligned}
\left|\left[b, I_{\gamma}\right]\left(f \chi_{B\left(y, r^{c}\right)}\right)\right| & \leq \sum_{k=0}^{\infty} \int_{2^{k+1} B \backslash 2^{k} B}\left|b(x)-b_{B}\right| \frac{|f(z)|}{|x-z|^{n-\gamma}} d z \\
& +\sum_{k=0}^{\infty} \int_{2^{k+1} B \backslash 2^{k} B}\left|b(z)-b_{B}\right| \frac{|f(z)|}{|x-z|^{n-\gamma}} d z \\
& =: J_{1}+J_{2}
\end{aligned}
$$

By Theorem 2,

$$
\begin{aligned}
& J_{1}=\left|b(x)-b_{B}\right| \sum_{k=0}^{\infty} \int_{2^{k+1} B \backslash 2^{k} B} \frac{|f(z)|}{|x-z|^{n-\gamma}} d z \\
& \leq\left|b(x)-b_{B}\right| \sum_{k=0}^{\infty}\left|2^{k+1} B\right|^{-1+\gamma / n} \int_{2^{k+1} B}|f(z)| d z \\
& \leq\left|b(x)-b_{B}\right| \sum_{k=0}^{\infty}\left|2^{k+1} B\right|^{\gamma / n} \inf _{x \in 2^{k+1} B} M f(x) \\
& \leq\left|b(x)-b_{B}\right| \sum_{k=0}^{\infty} \frac{\left\|\chi_{2^{k+1} B}\right\|_{\left(L^{\vec{p}}, L^{\vec{s}}\right)^{\alpha}}}{\left\|\chi_{2^{k+1} B}\right\|_{\left(L^{\vec{p}}, L^{\vec{s}}\right)^{\alpha}}}\left|2^{k+1} B\right|^{\gamma / n} \inf _{x \in 2^{k+1} B} M f(x) \\
& \leq\left|b(x)-b_{B}\right|\|f\|_{\left(L^{\vec{p}}, L^{\vec{s}}\right)^{\alpha}} \sum_{k=0}^{\infty}\left|2^{k+1} B\right|^{\gamma / n-1 / \alpha} \\
& \lesssim|B|^{\gamma / n-1 / \alpha}\|f\|_{\left(L^{\left.\vec{p}, L^{\vec{s}}\right)^{\alpha}} \mid\right.}\left|b(x)-b_{B}\right| .
\end{aligned}
$$


By Hölder's inequality, we have

$$
\begin{aligned}
J_{2} & \leq \sum_{k=0}^{\infty} \int_{2^{k+1} B \backslash 2^{k} B}\left|b(z)-b_{B}\right| \frac{|f(z)|}{|x-z|^{n-\alpha}} d z \\
& \leq \sum_{k=0}^{\infty}\left|2^{k+1} B\right|^{-1+\frac{\alpha}{n}} \int_{2^{k+1} B \backslash 2^{k} B}\left|b(z)-b_{B}\right||f(z)| d z \\
& \leq \sum_{k=0}^{\infty}\left|2^{k+1} B\right|^{\frac{\alpha}{n}}\left(\frac{1}{\left|2^{k+1} B\right|} \int_{2^{k+1} B}\left|b(z)-b_{B}\right|^{t^{\prime}} d z\right)^{\frac{1}{t^{\prime}}}\left(\frac{1}{\left|2^{k+1} B\right|} \int_{2^{k+1} B}|f(z)|^{t} d z\right)^{\frac{1}{t}},
\end{aligned}
$$

where $1<t<\min \left\{p_{1}, p_{2}, \cdots, p_{n}\right\}$. From Lemma 6 ,

$$
J_{2} \lesssim \sum_{k=0}^{\infty}\left|2^{k+1} B\right|^{\frac{\alpha}{n}} k\|b\|_{B M O} \inf _{x \in 2^{k+1} B} M_{t} f(x) .
$$

Similar to $J_{1}$, we have

$$
J_{2} \lesssim|B|^{\gamma / n-1 / \alpha}\|b\|_{B M O}\|f\|_{\left(L^{\left.\vec{p}, L^{\vec{s}}\right)^{\alpha}}\right.} .
$$

Now, we can say $\left[b, I_{\alpha}\right] f$ are well defined.

\section{The Boundedness of $I_{\gamma}$}

In this section, we will prove the conclusions of Theorem 3.

Proof of Theorem 3. By Remark 5, we only need to prove the boundedness of $I_{\gamma}$ on mixednorm amalgam spaces if $\gamma=\frac{n}{\alpha}-\frac{n}{\beta}$. Let $f \in\left(L^{\vec{p}}, L^{\vec{s}}\right)^{\alpha}\left(\mathbb{R}^{n}\right), B=B(y, r)$, and

$$
f=f_{1}+f_{2}=f \chi_{2 B}+f \chi_{(2 B)} .
$$

where $\chi_{2 B}$ is the characteristic function of $2 B$. By the linearity of the fractional integral operator $I_{\gamma}$, one can write

$$
\begin{aligned}
& |B(y, r)|^{\frac{1}{\beta}-\frac{1}{n} \sum_{i=1}^{n} \frac{1}{q_{i}}-\frac{1}{n} \sum_{i=1}^{n} \frac{1}{s_{i}}}\left\|I_{\alpha}(f) \chi_{B(y, r)}\right\|_{L^{\vec{q}}}
\end{aligned}
$$

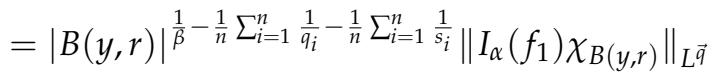

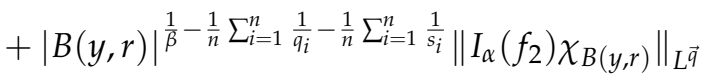

$$
\begin{aligned}
& :=\mathrm{I}(y, r)+\mathrm{II}(y, r)
\end{aligned}
$$

Below, we will give the estimates of $I(y, r)$ and $I I(y, r)$, respectively. By the $\left(L^{\vec{p}}, L^{\vec{q}}\right)$ boundedness of $I_{\gamma}$ (see Lemma 1),

$$
\begin{aligned}
\mathrm{I}(y, r) & \leq|B(y, r)|^{\frac{1}{\beta}-\frac{1}{n} \sum_{i=1}^{n} \frac{1}{q_{i}}-\frac{1}{n} \sum_{i=1}^{n} \frac{1}{s_{i}}}\left\|f \chi_{2 B(y, r)}\right\|_{L^{\vec{p}}} \\
& \sim|2 B(y, r)|^{\frac{1}{\alpha}-\frac{1}{n} \sum_{i=1}^{n} \frac{1}{p_{i}}-\frac{1}{n} \sum_{i=1}^{n} \frac{1}{s_{i}}}\left\|f \chi_{2 B(y, r)}\right\|_{L^{\vec{p}} .}
\end{aligned}
$$

Thus,

$$
\sup _{r>0}\|\mathrm{I}(\cdot, r)\|_{L^{\vec{s}}} \lesssim\|f\|_{\left(L^{\vec{p}}, L^{\vec{s}}\right)^{\alpha}}
$$


Let us now turn to the estimate of $\mathrm{II}(y, r)$. First, it is clear that when $x \in B(y, r)$ and $z \in(2 B)^{c}$, we obtain $|x-z| \sim|y-z|$. Then, we decompose $\mathbb{R}^{n}$ into a geometrically increasing sequence of concentric balls and obtain the following pointwise estimate:

$$
\begin{aligned}
I_{\gamma}\left(f_{2}\right)(x) & =\int_{(2 B)^{c}} \frac{|f(z)|}{|x-z|^{n-\gamma}} d z \\
& \sim \sum_{j=1}^{\infty} \int_{2^{j+1} B \backslash 2^{j} B} \frac{|f(z)|}{|y-z|^{n-\gamma}} d z \\
& \lesssim \sum_{j=1}^{\infty} \frac{1}{\left|2^{j+1} B\right|^{1-\frac{\gamma}{n}}} \int_{2^{j+1} B}|f(z)| d z .
\end{aligned}
$$

Combining (14) and Hölder's inequality, we obtain

$$
\begin{aligned}
\mathrm{II} & =|B(y, r)|^{\frac{1}{\beta}-\frac{1}{n} \sum_{i=1}^{n} \frac{1}{q_{i}}-\frac{1}{n} \sum_{i=1}^{n} \frac{1}{s_{i}}}\left\|\chi_{B(y, r)} \int_{R^{n}} \frac{\left|f_{2}(z)\right|}{|\cdot-z|^{n-\gamma}} d z\right\|_{L^{\vec{q}}} \\
& \lesssim|B(y, r)|^{\frac{1}{\beta}-\frac{1}{n} \sum_{i=1}^{n} \frac{1}{s_{i}}} \sum_{j=1}^{\infty} \frac{1}{\left|2^{j+1} B(y, r)\right|^{1-\frac{\gamma}{n}}} \int_{2^{j+1} B(y, r)}|f(z)| d z \\
& \leq \sum_{j=1}^{\infty} 2^{-j\left(\frac{1}{\beta}-\frac{1}{n} \sum_{i=1}^{n} \frac{1}{s_{i}}\right)}\left|2^{j+1} B(y, r)\right|^{\frac{1}{\alpha}-\frac{1}{n} \sum_{i=1}^{n} \frac{1}{s_{i}}-\frac{1}{n} \sum_{i=1}^{n} \frac{1}{p_{i}}}\left\|f \chi_{2^{j+1} B(y, r)}\right\|_{L^{\vec{p}}}
\end{aligned}
$$

where $\frac{1}{\alpha}=\frac{\gamma}{n}+\frac{1}{\beta}$. By $\frac{1}{\beta}-\frac{1}{n} \sum_{i=1}^{n} \frac{1}{s_{i}}>0$,

$$
\sum_{j=1}^{\infty} 2^{-j\left(\frac{1}{\beta}-\frac{1}{n} \sum_{i=1}^{n} \frac{1}{s_{i}}\right)} \sim 1
$$

Thus,

$$
\sup _{r>0}\|\mathrm{II}(\cdot, r)\|_{L^{\vec{s}}} \lesssim\|f\|_{\left(L^{\vec{p}}, L^{\vec{s}}\right)^{\alpha}} \cdot
$$

Therefore, using (13) and (15),

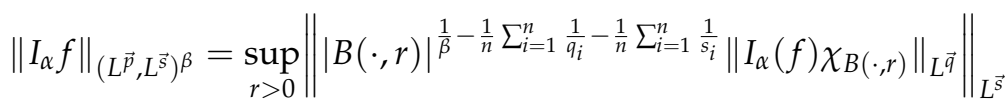

$$
\begin{aligned}
& \leq \sup _{r>0}\|\mathrm{I}(\cdot, r)\|_{L^{\vec{s}}}+\sup _{r>0}\|\mathrm{II}(\cdot, r)\|_{L^{\vec{s}}} \\
& \lesssim\|f\|_{\left(L^{\vec{p}}, L^{\vec{S}}\right)^{\alpha}} \text {. }
\end{aligned}
$$

The proof is completed.

Let $0<\gamma<n$. The related fractional maximal function is defined as

$$
M_{\gamma} f(x):=\sup _{B \ni x} \frac{1}{|B|^{1-\frac{\gamma}{n}}} \int_{B}|f(y)| d y,
$$

where the supremum is taken over all cubes $B \subset \mathbb{R}^{n}$ containing $x$. It is well known that

$$
\left|M_{\gamma} f(x)\right| \lesssim I_{\gamma}(|f|)(x) .
$$

An immediate application of the above inequality (16) is the following strong-type for the operators $M_{\gamma}$.

Corollary 2. Let $0<\gamma<n, 1<\vec{p}, \vec{q}<\infty, 1<\vec{s} \leq \infty, \frac{1}{n} \sum_{i=1}^{n} \frac{1}{s_{i}} \leq \frac{1}{\alpha} \leq \frac{1}{n} \sum_{i=1}^{n} \frac{1}{p_{i}}$, and $\frac{1}{n} \sum_{i=1}^{n} \frac{1}{s_{i}} \leq \frac{1}{\beta} \leq \frac{1}{n} \sum_{i=1}^{n} \frac{1}{q_{i}}$. Assume that $\gamma=\sum_{i=1}^{n} \frac{1}{p_{i}}-\sum_{i=1}^{n} \frac{1}{q_{i}}=\frac{n}{\alpha}-\frac{n}{\beta}$. Then, the fractional integral operators $M_{\gamma}$ are bounded from $\left(L^{\vec{p}}, L^{\vec{s}}\right)^{\alpha}\left(\mathbb{R}^{n}\right)$ to $\left(L^{\vec{q}}, L^{\vec{s}}\right)^{\beta}\left(\mathbb{R}^{n}\right)$. 
Before the following corollary, let us recall generalized fractional integral operators.

Suppose that $\mathcal{L}$ are linear operators which generate an analytic semigroup $\left\{e^{-t \mathcal{L}}\right\}_{t>0}$ on $L^{2}\left(\mathbb{R}^{n}\right)$ with a kernel $p_{t}(x, y)$ satisfying

$$
\left|p_{t}(x, y)\right| \leq \frac{C_{1}}{t^{n / 2}} e^{-C_{2} \frac{|x-y|^{2}}{t}} x, y \in \mathbb{R}^{n},
$$

where $C_{1}, C_{2}>0$ are independent of $x, y$ and $t$.

For any $0<\gamma<n$, the generalized fractional integral operators $\mathcal{L}^{-\gamma / 2}$ associated with the operator $\mathcal{L}$ are defined by

$$
\mathcal{L}^{-\gamma / 2} f(x)=\frac{1}{\Gamma(\gamma / 2)} \int_{0}^{\infty} e^{-t \mathcal{L}}(f)(x) \frac{d t}{t^{-\gamma / 2+1}} .
$$

Note that if $\mathcal{L}=-\Delta$ is the Laplacian on $\mathbb{R}^{n}$, then $\mathcal{L}^{-\gamma / 2}$ is the classical fractional integral operators $I_{\gamma}$. See, for example, [28] (Chapter 5). By the Gaussian upper bound of kernel $p_{t}(x, y)$, for all $x \in \mathbb{R}^{n}$,

$$
\left|\mathcal{L}^{-\gamma / 2} f(x)\right| \leq C I_{\gamma}(|f|)(x) .
$$

(see [36]). In fact, if we denote the the kernel of $\mathcal{L}^{-\gamma / 2}$ by $K_{\gamma}(x, y)$, then

$$
\begin{aligned}
\mathcal{L}^{-\gamma / 2} f(x) & =\frac{1}{\Gamma(\gamma / 2)} \int_{0}^{\infty} e^{-t \mathcal{L}}(f)(x) \frac{d t}{t^{-\gamma / 2+1}} \\
& =\frac{1}{\Gamma(\gamma / 2)} \int_{0}^{\infty} \int_{\mathbb{R}^{n}} p_{t}(x, y) f(y) d y \frac{d t}{t^{-\gamma / 2+1}} \\
& =\int_{\mathbb{R}^{n}} \frac{1}{\Gamma(\gamma / 2)} \int_{0}^{\infty} p_{t}(x, y) \frac{d t}{t^{-\gamma / 2+1}} \cdot f(y) d y \\
& =\int_{\mathbb{R}^{n}} K_{\gamma}(x, y) \cdot f(y) d y .
\end{aligned}
$$

Hence, by the Gaussian upper bound,

$$
\begin{aligned}
\left|K_{\gamma}(x, y)\right| & =\left|\frac{1}{\Gamma(\gamma / 2)} \int_{0}^{\infty} p_{t}(x, y) \frac{d t}{t^{-\gamma / 2+1}}\right| \\
& \leq \frac{1}{\Gamma(\gamma / 2)} \int_{0}^{\infty}\left|p_{t}(x, y)\right| \frac{d t}{t^{-\gamma / 2+1}} \\
& \leq C \int_{0}^{\infty} e^{-C_{2} \frac{|x-y|^{2}}{t}} \frac{d t}{t^{n / 2-\gamma / 2+1}} \\
& \leq C \cdot \frac{1}{|x-y|^{n-\gamma}} .
\end{aligned}
$$

Considering the pointwise inequality (17), as a consequence of Theorem 2, we have the following corollary.

Corollary 3. Let $0<\gamma<n, 1<\vec{p}, \vec{q}<\infty, 1<\vec{s} \leq \infty, \frac{1}{n} \sum_{i=1}^{n} \frac{1}{s_{i}} \leq \frac{1}{\alpha} \leq \frac{1}{n} \sum_{i=1}^{n} \frac{1}{p_{i}}$, and $\frac{1}{n} \sum_{i=1}^{n} \frac{1}{s_{i}} \leq \frac{1}{\beta} \leq \frac{1}{n} \sum_{i=1}^{n} \frac{1}{q_{i}}$. Assume that $\gamma=\sum_{i=1}^{n} \frac{1}{p_{i}}-\sum_{i=1}^{n} \frac{1}{q_{i}}=\frac{n}{\alpha}-\frac{n}{\beta}$. Then the generalized fractional integral operators $\mathcal{L}^{\gamma / 2}$ are bounded from $\left(L^{\vec{p}}, L^{\vec{s}}\right)^{\alpha}\left(\mathbb{R}^{n}\right)$ to $\left(L^{\vec{q}}, L^{\vec{s}}\right)^{\beta}\left(\mathbb{R}^{n}\right)$.

\section{The Boundedness of $\left[b, I_{\gamma}\right]$}

In this section, we show the proof of Theorem 3.

Proof of Theorem 4. Let $f \in\left(L^{\vec{p}}, L^{\vec{s}}\right)^{\alpha}\left(\mathbb{R}^{n}\right), B=B(y, r)$, and

$$
f=f_{1}+f_{2}=f \chi_{2 B}+f \chi_{(2 B)} .
$$


By the linearity of the commutator operators $\left[b, I_{\gamma}\right]$, we write

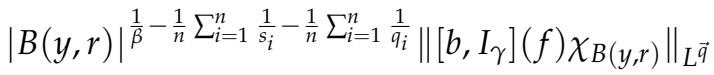

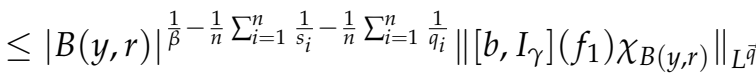

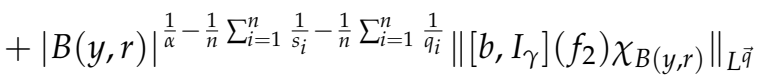

$$
\begin{aligned}
& :=\mathrm{I}(y, r)+\mathrm{II}(y, r) \text {. }
\end{aligned}
$$

By Lemma 2 and observing that $\frac{1}{\beta}-\frac{1}{n} \sum_{i=1}^{n} \frac{1}{q_{i}}=\frac{1}{\alpha}-\frac{1}{n} \sum_{i=1}^{n} \frac{1}{p_{i}}$,

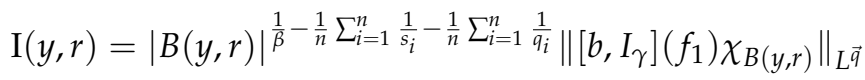

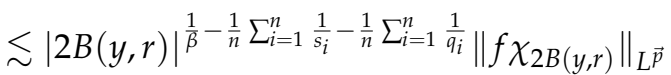

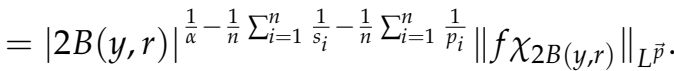

Thus,

$$
\sup _{r>0}\|I(y, r)\|_{L^{\vec{s}}} \lesssim\|f\|_{\left(L^{\vec{p}}, L^{\vec{s}}\right)^{\alpha}}
$$

Now, let us turn to the estimate of $\operatorname{II}(y, r)$. By the definition of $\left[b, I_{\gamma}\right]$, we have

$$
\left|\left[b, I_{\gamma}\right]\left(f_{2}\right)(x)\right| \leq\left|b(x)-b_{B(y, r)}\right| \cdot\left|I_{\gamma}\left(f_{2}\right)(x)\right|+\left|I_{\gamma}\left[\left(b_{B(y, r)}-b\right) f_{2}\right](x)\right| .
$$

Therefore,

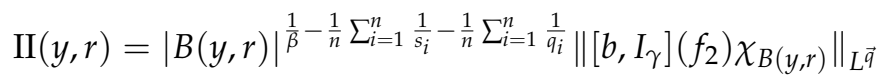

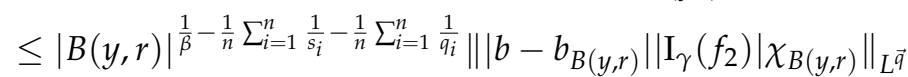

$$
\begin{aligned}
& +\left\|I_{\gamma}\left[\left(b_{B(y, r)}-b\right) f_{2}\right] \chi_{B(y, r)}\right\|_{L^{\vec{q}}} \\
& \leq|B(y, r)|^{\frac{1}{\beta}-\frac{1}{n} \sum_{i=1}^{n} \frac{1}{s_{i}}-\frac{1}{n} \sum_{i=1}^{n} \frac{1}{g_{i}}[}\left[|| b-b_{B(y, r)}|| I_{\gamma}\left(f_{2}\right) \mid \chi_{B(y, r)} \|_{L^{\vec{q}}}\right.
\end{aligned}
$$

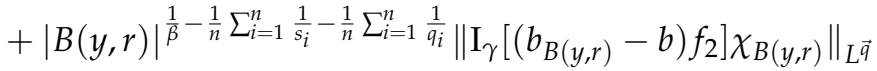

$$
\begin{aligned}
& :=\mathrm{II}_{1}(y, r)+\mathrm{II}_{2}(y, r) \text {. }
\end{aligned}
$$

By (14), Lemma 5(ii), and Hölder's inequality,

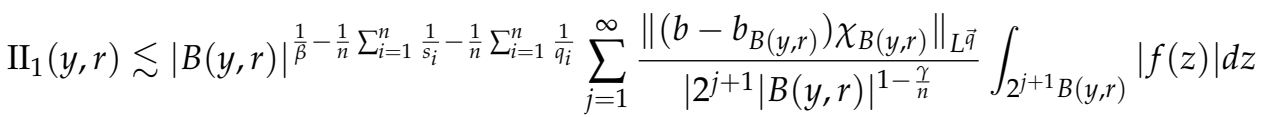

$$
\begin{aligned}
& \sim\|b\|_{B M O} \sum_{j=1}^{\infty}|B(y, r)|^{\frac{1}{\beta}-\frac{1}{n} \sum_{i=1}^{n} \frac{1}{s_{i}}} \frac{1}{\left|2^{j+1} B(y, r)\right|^{1-\frac{\gamma}{n}}} \int_{2^{j+1} B(y, r)}|f(z)| d z \\
& =\|b\|_{B M O} \sum_{j=1}^{\infty} 2^{-j\left(\frac{1}{\beta}-\frac{1}{n} \sum_{i=1}^{n} \frac{1}{s_{i}}\right)}\left|2^{j+1} B(y, r)\right|^{\frac{1}{\alpha}-\frac{1}{n} \sum_{i=1}^{n} \frac{1}{s_{i}}-\frac{1}{n} \sum_{i=1}^{n} \frac{1}{p_{i}}}\left\|f \chi_{2^{j+1} B(y, r)}\right\|_{L^{\vec{p}}} .
\end{aligned}
$$

Due to the assumption $\frac{1}{\beta}-\frac{1}{n} \sum_{i=1}^{n} \frac{1}{s_{i}}>0$,

$$
\sum_{j=1}^{\infty} 2^{-j\left(\frac{1}{\beta}-\frac{1}{n} \sum_{i=1}^{n} \frac{1}{s_{i}}\right)} \sim 1
$$

Thus,

$$
\sup _{r>0}\|\mathrm{I}(y, r)\|_{L^{\vec{s}}\left(\mathbb{R}^{n}\right)} \lesssim\|b\|_{B M O}\|f\|_{\left(E^{\vec{p}}, L^{\vec{s}}\right)^{\alpha}\left(\mathbb{R}^{n}\right)} .
$$


For the estimates of $\mathrm{II}_{2}(y, r)$, by (14), we have

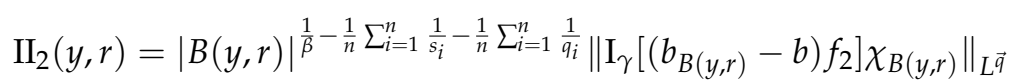

$$
\begin{aligned}
& \leq|B(y, r)|^{\frac{1}{\beta}-\frac{1}{n} \sum_{i=1}^{n} \frac{1}{s_{i}}} \cdot \sum_{j=1}^{+\infty} \frac{1}{\left|2^{j+1} B(y, r)\right|^{1-\frac{\gamma}{n}}} \int_{2^{j+1} B(y, r)}|f(z)|\left|b(z)-b_{B(y, r)}\right| d z \\
& \leq|B(y, r)|^{\frac{1}{\beta}-\frac{1}{n} \sum_{i=1}^{n} \frac{1}{s_{i}}} \cdot \sum_{j=1}^{+\infty} \frac{1}{\left|2^{j+1} B(y, r)\right|^{1-\frac{\gamma}{n}}} \int_{2^{j+1} B(y, r)}|f(z)|\left|b(z)-b_{2^{j+1} B(y, r)}\right| d z \\
& +|B(y, r)|^{\frac{1}{\beta}-\frac{1}{n} \sum_{i=1}^{n} \frac{1}{s_{i}}} \cdot \sum_{j=1}^{+\infty} \frac{1}{\left|2^{j+1} B(y, r)\right|^{1-\frac{\gamma}{n}}} \int_{2^{j+1} B(y, r)}|f(z)| d z \cdot\left|b_{2^{j+1} B(y, r)}-b_{B(y, r)}\right| \\
& =: \mathrm{II}_{21}(y, r)+\mathrm{II}_{22}(y, r) \text {. }
\end{aligned}
$$

To estimate $\mathrm{II}_{21}(y, r)$, applying Hölder's inequality and Lemma 6(ii), we can deduce that

$$
\begin{aligned}
& \mathrm{II}_{21}(y, r) \leq|B(y, r)|^{\frac{1}{\beta}-\frac{1}{n} \sum_{i=1}^{n} \frac{1}{s_{i}}} \cdot \sum_{j=1}^{+\infty} \frac{\left\|\left(b-b_{2^{j+1} B(y, r)}\right) \chi_{2^{j+1} B(y, r)}\right\|_{L^{\vec{p}^{\prime}}}}{\left|2^{j+1} B(y, r)\right|^{1-\frac{\gamma}{n}}}\left\|f \chi_{2^{j+1} B(y, r)}\right\|_{L^{\vec{p}}} \\
& \sim\|b\|_{B M O} \sum_{j=1}^{+\infty} 2^{j\left(\frac{1}{\beta}-\frac{1}{n} \sum_{i=1}^{n} \frac{1}{s_{i}}\right)} \cdot\left|2^{j+1} B(y, r)\right|^{\frac{1}{\alpha}-\frac{1}{n} \sum_{i=1}^{n} \frac{1}{s_{i}}-\frac{1}{n} \sum_{i=1}^{n} \frac{1}{p_{i}}}\left\|f \chi_{2^{j+1} B(y, r)}\right\|_{L^{\vec{p}}},
\end{aligned}
$$

where $\frac{1}{\alpha}=\frac{1}{\beta}+\frac{\gamma}{n}$. By (8), we obtain

$$
\sup _{r>0}\left\|\mathrm{II}_{21}(y, r)\right\|_{L^{\vec{s}}} \lesssim\|b\|_{B M O} \cdot\|f\|_{\left(L^{\vec{p}}, L^{\vec{S}}\right)^{\alpha}}
$$

Now, we estimate $\mathrm{II}_{22}(y, r)$. An application of Lemma 5(i) and Hölder's inequality gives us that

$$
\begin{aligned}
\mathrm{II}_{22}(y, r) & \lesssim\|b\|_{B M O} \sum_{j=1}^{+\infty} \frac{j|B(y, r)|^{\frac{1}{\beta}-\frac{1}{n} \sum_{i=1}^{n} \frac{1}{s_{i}}}}{\left|2^{j+1} B(y, r)\right|^{1-\frac{\gamma}{n}}} \int_{2^{j+1} B(y, r)}|f(z)| d z \\
& \leq\|b\|_{B M O} \sum_{j=1}^{+\infty} \frac{j}{2^{j\left(\frac{1}{\beta}-\frac{1}{n} \sum_{i=1}^{n} \frac{1}{s_{i}}\right)}} \cdot\left|2^{j+1} B(y, r)\right|^{\frac{1}{\alpha}-\frac{1}{n} \sum_{i=1}^{n} \frac{1}{s_{i}}-\frac{1}{n} \sum_{i=1}^{n} \frac{1}{p_{i}}}\left\|f \chi_{2^{j+1} B(y, r)}\right\|_{L^{\vec{p}}} .
\end{aligned}
$$

By (19), we obtain

$$
\sup _{r>0}\left\|\mathrm{II}_{22}(y, r)\right\|_{L^{\vec{s}}} \leq\|b\|_{B M O} \cdot\|f\|_{\left(L^{\vec{p}}, L^{\vec{s}}\right)^{\alpha}} .
$$

Combining (18), (20)-(22), we conclude that

$$
\begin{aligned}
\left\|I_{\gamma}(f)\right\|_{\left(L^{\vec{p}}, L^{\vec{s}}\right)^{\alpha}} & \leq \sup _{r>0}\|\mathrm{I}(\cdot, r)\|_{L^{\vec{s}}}+\sup _{r>0}\|\mathrm{II}(\cdot, r)\|_{L^{\vec{s}}} \\
& \leq \sup _{r>0}\|\mathrm{I}(\cdot, r)\|_{L^{\vec{s}}}+\sup _{r>0}\left\|\mathrm{II}_{1}(\cdot, r)\right\|_{L^{\vec{s}}}+\sup _{r>0}\left\|\mathrm{II}_{2}(\cdot, r)\right\|_{L^{\vec{s}}} \\
& \leq \sup _{r>0}\|\mathrm{I}(\cdot, r)\|_{L^{\vec{s}}}+\sup _{r>0}\left\|\mathrm{II}_{1}(\cdot, r)\right\|_{L^{\vec{s}}}+\sup _{r>0}\left\|\mathrm{II}_{21}(\cdot, r)\right\|_{L^{\vec{s}}}+\sup _{r>0}\left\|\mathrm{II}_{22}(\cdot, r)\right\|_{L^{\vec{s}}} \\
& \leq\|b\|_{B M O} \cdot\|f\|_{\left(L^{\vec{p}}, L^{\vec{s}}\right)^{\alpha}} .
\end{aligned}
$$

The proof is completed.

\section{A Characterization of $B M O$}

In this section, we prove Theorem 5 . As the consequence of Theorems 4 and 5, the characterization of $B M O\left(\mathbb{R}^{n}\right)$, Corollary 1, is proved. 
Proof of Theorem 5. Assume that $\left[b, I_{\alpha}\right]$ is bounded from $\left(L^{\vec{p}}, L^{\vec{s}}\right)^{\alpha}\left(\mathbb{R}^{n}\right)$ to $\left(L^{\vec{q}}, L^{\vec{s}}\right)^{\beta}\left(\mathbb{R}^{n}\right)$. We use the same method as Janson [37]. Choose $0 \neq z_{0} \in \mathbb{R}^{n}$ such that $0 \notin B\left(z_{0}, 2\right)$. Then for $x \in B\left(z_{0}, 2\right),|x|^{n-\alpha} \in C^{\infty}\left(B\left(z_{0}, 2\right)\right)$. Hence, $|x|^{n-\alpha}$ can be written as the absolutely convergent Fourier series:

$$
|x|^{n-\alpha} \chi_{B\left(z_{0}, 2\right)}(x)=\sum_{m \in \mathbb{Z}^{n}} a_{m} e^{2 i m \cdot x} \chi_{B\left(z_{0,2}\right)}(x)
$$

with $\sum_{m \in \mathbb{Z}^{n}}\left|a_{m}\right|<\infty$.

For any $x_{0} \in \mathbb{R}^{n}$ and $t>0$, let $B=B\left(x_{0}, t\right)$ and $B_{z_{0}}=B\left(x_{0}+z_{0} t, t\right)$. Let $s(x)=$ $\overline{\operatorname{sgn}\left(\int_{B_{z_{0}}}(b(x)-b(y)) d y\right)}$. Then,

$$
\frac{1}{|B|} \int_{B}\left|b(x)-b_{B_{z_{0}}}\right|=\frac{1}{|B|} \frac{1}{\left|B_{z_{0}}\right|} \int_{B} \int_{B_{z_{0}}} s(x)(b(x)-b(y)) d y d x .
$$

If $x \in B$ and $y \in B_{z_{0}}$, then $\frac{y-x}{t} \in B\left(z_{0}, 2\right)$. Thereby,

$$
\begin{aligned}
& \frac{1}{|B|} \int_{B}\left|b(x)-b_{B_{z_{0}}}\right| \\
= & t^{-n-\gamma} \int_{B} \int_{B_{z_{0}}} s(x)(b(x)-b(y))|x-y|^{\alpha-n}\left(\frac{|x-y|}{t}\right)^{n-\gamma} d y d x \\
= & t^{-n-\gamma} \sum_{m \in \mathbb{Z}^{n}} a_{m} \int_{B} \int_{B_{z_{0}}} s(x)(b(x)-b(y))|x-y|^{\gamma-n} e^{-2 i m \cdot \frac{y}{t}} d y \times e^{2 i m \cdot \frac{x}{t}} d x \\
= & t^{-n-\gamma} \sum_{m \in \mathbb{Z}^{n}} a_{m} \int_{B}\left[b, I_{\gamma}\right]\left(e^{-2 i m \cdot \dot{t}} \chi_{B_{z_{0}}}\right)(x) \times s(x) e^{2 i m \cdot \frac{x}{t}} d x .
\end{aligned}
$$

By (5) and Proposition 5,

$\frac{1}{|B|} \int_{B}\left|b(x)-b_{B_{z_{0}}}\right| \lesssim t^{-n-\gamma} \sum_{m \in \mathbb{Z}^{n}} a_{m}\left\|\left[b, I_{\gamma}\right]\left(e^{-2 i m \cdot \dot{\bar{t}}} \chi_{B_{z_{0}}}\right)\right\|_{\left(L^{\natural}, L^{\vec{b}}\right)^{\beta}}\left\|S \cdot e^{-2 i m \cdot \dot{\bar{t}}} \chi_{B}\right\|_{\mathcal{H}\left(\vec{q}^{\prime}, \vec{s}^{\prime}, \beta^{\prime}\right)} \cdot$

By calculation,

$$
\left\|S \cdot e^{-2 i m \cdot \dot{\bar{t}}} \chi_{B}\right\|_{\mathcal{H}\left(\vec{q}^{\prime}, \vec{s}^{\prime}, \beta^{\prime}\right)} \lesssim t^{n / \beta^{\prime}}
$$

Hence,

$$
\frac{1}{|B|} \int_{B}\left|b(x)-b_{B_{z_{0}}}\right| \lesssim t^{-n-\gamma+n / \beta^{\prime}} \sum_{m \in \mathbb{Z}^{n}} a_{m}\left\|\left[b, I_{\gamma}\right]\left(e^{-2 i m \cdot \dot{\dot{t}}} \chi_{B_{z_{0}}}\right)\right\|_{\left(L^{\vec{q}}, L^{\vec{s}}\right)^{\beta}} .
$$

According to the hypothesis

$$
\begin{aligned}
\frac{1}{|B|} \int_{B}\left|b(x)-b_{B_{z_{0}}}\right| & \lesssim t^{-n-\gamma+n / \beta^{\prime}}\left\|\left[b, I_{\gamma}\right]\right\| \sum_{m \in \mathbb{Z}^{n}} a_{m}\left\|e^{-2 i m \cdot \dot{\epsilon}} \chi_{B_{z_{0}}}\right\|_{\left(L^{\vec{p}}, L^{\vec{s}}\right)^{\alpha}} \\
& \leq t^{-n-\gamma+n / \beta^{\prime}+n / \alpha}\left\|\left[b, I_{\gamma}\right]\right\| \sum_{m \in \mathbb{Z}^{n}} a_{m} \\
& \lesssim\left\|\left[b, I_{\gamma}\right]\right\| .
\end{aligned}
$$

Thus, we have

$$
\frac{1}{|B|} \int_{B}|b(x)-b(y)| d x \leq \frac{2}{|Q|} \int_{B}\left|b(x)-b_{B_{z_{0}}}\right| d x \lesssim\left\|\left[b, I_{\gamma}\right]\right\| .
$$

Hence $b \in B M O\left(\mathbb{R}^{n}\right)$. 
Author Contributions: Writing-review and editing, H.Z.; writing-review and editing, H.Z. and J.Z.; funding acquisition, J.Z. All authors have read and agreed to the published version of the manuscript.

Funding: This research was funded by the National Natural Science Foundation of China (Grant No. 12061069).

Institutional Review Board Statement: Not applicable.

Informed Consent Statement: Not applicable.

Data Availability Statement: Not applicable.

Acknowledgments: All authors would like to express their thanks to the referees for valuable advice regarding previous versions of this paper.

Conflicts of Interest: The authors declare no conflict of interest.

\section{References}

1. Benedek, A.; Panzone, R. The space $L^{p}$, with mixed norm. Duke Math. 1961, 28, 301-324. [CrossRef]

2. Antonic, N.; Ivec, I. On the Hörmander-Mihlin theorem for mixed-norm Lebesgue spaces. Math. Anal. Appl. 2016, 433, 176-199. [CrossRef]

3. Kim, D. Elliptic and parabolic equations with measurable coefficients in $L^{p}$-spaces with mixed norms. Methods Appl. Anal. 2008, 15, 437-468. [CrossRef]

4. Krylov, N.V. Parabolic equations with VMO coefficients in Sobolev spaces with mixed norms. Funct. Anal. 2007, 250, 521-558. [CrossRef]

5. Fernandez, D.L. Lorentz spaces, with mixed norms. Funct. Anal. 1977, 25, 128-146. [CrossRef]

6. Milman, M. Embeddings of Lorentz-Marcinkiewicz spaces with mixed norms. Anal. Math. 1978, 4, 215-223. [CrossRef]

7. Milman, M. A note on L $(\mathrm{p}, \mathrm{q})$ spaces and Orlicz spaces with mixed norms. Proc. Am. Math. Soc. 1981, 83, 743-746. [CrossRef]

8. Cleanthous, G.; Georgiadis, A.G.; Nielsen, M. Anisotropic mixed-norm Hardy spaces. J. Geom. Anal. 2017, 27, 2758-2787 [CrossRef]

9. Besov, O.V.; Il'in, V.P.; Nikolski1, S.M. Integral Representations of Functions, and Embedding Theorems, 2nd ed.; Fizmatlit "Nauka"; V.H. Winston: Moscow, Russian, 1996; p. 480.

10. Nogayama, T. Mixed Morrey spaces. Positivity 2019, 23, 961-1000. [CrossRef]

11. Nogayama, T. Boundedness of commutators of fractional integral operators on mixed Morrey spaces. Integral Transform. Spec. Funct. 2019, 30, 790-816. [CrossRef]

12. Chen, T.; Sun, W. Iterated and Mixed Weak Norms with Applications to Geometric Inequalities. arXiv 2017, arXiv:1712.01064.

13. Huang, L.; Yang, D. On Function Spaces with Mixed Norms-A Survey. arXiv 2019, arXiv:1908.03291.

14. Morrey, C.B. On the solutions of quasi-linear elliptic partial differential equations. Trans. Am. Math. Soc. 1938, 43, 126-166. [CrossRef]

15. Burenkov, V.; Guliyev, H.V. Necessary and sufficient conditions for boundedness of the maximal operator in the local Morrey-type spaces. Studia Math. 2004, 163, 157-176. [CrossRef]

16. Zhang, H.; Zhou, J. The Boundedness of Fractional Integral Operators in Local and Global Mixed Morrey-type Spaces. arXiv 2021, arXiv:2102.01304v1.

17. Zhao, J.; Kosti, M.; Du, W.S.; On Generalizations of Sampling Theorem and Stability Theorem in Shift-Invariant Subspaces of Lebesgue and Wiener Amalgam Spaces with Mixed-Norms. Symmetry 2021, 13, 331. [CrossRef]

18. Wiener, N. On the representation of functions by trigonometrical integrals. Math. Z. 1926, 24, 575-616 [CrossRef]

19. Hollandf. Harmonic analysis on amalgams of $L^{p}$ and $l 9$. J. Lond. Math. Soc. 1975, 10, 295-305.

20. Fournier, J.J.F.; Stewart J. Amalgams of $L^{p}$ and 19 . B Am. Math. Soc. 1985, 13, 1-22. [CrossRef]

21. Feichtinger, H.G. Generalized amalgams and its applications to Fourier transforms. Can. J. Math. 1990, 42, 395-409. [CrossRef]

22. Feichtinger, H.G. Wiener amalgams over Euclidean spaces and some of their applications. In Function Spaces; Lecture Notes in Pure and Appl. Math.; CRC Press: Boca Raton, FL, USA, 2020.

23. Feuto, J.; Fofana, I.; Koua, K. Integrable fractional mean functions on spaces of homogeneous type. Afr. Diaspora J. Math. 2010, 9, 8-30.

24. Cordero, E.; Nicola, F. Metaplectic representation on Wiener amalgam spaces and applications to the Schrödinger equation. J. Funct. Anal. 2008, 254, 506-534. [CrossRef]

25. Fofana, I. Étude d'une classe d'espaces de fonctions contenant les espaces de Lorentz. Afr. Mat. 1988, 2, 29-50.

26. Gurkanl1, A.T. The Amalgam Spaces $W\left(L^{p(x)}, \ell^{\{p n\}}\right)$ and Boundedness of Hardy-Littlewood Maximal Operators. In Current Trends in Analysis and Its Applications. Trends in Mathematics; Mityushev, V., Ruzhansky, M., Eds.; Birkhauser: Basel, Switzerland, 2015. [CrossRef]

27. Zhang, Y.; Yang, D.; Yuan, W.; Wang, S. Real-variable characterizations of Orlicz-slice Hardy spaces. Anal. Appl. 2019, 17, 597-664. [CrossRef] 
28. Stein, E.M. Singular Integrals and Differentiability Properties of Functions; Princeton University Press: Princeton, NJ, USA, 1970.

29. Chanillo, S. A note on commutators. Indiana Univ. Math. 1982, 31, 7-16. [CrossRef]

30. Komori, Y. The Factorization of $H^{p}$ and the Commutators. Tokyo J. Math. 1983, 06, 435-445. [CrossRef]

31. Zhang, H.; Zhou, J. Necessary and sufficient conditions for boundedness of commutators of fractional integral operators on mixed-norm Lebesgue spaces. arXiv 2021, arXiv:2106.04926.

32. Wang, H. Estimates for Fractional Integral Operators and Linear Commutators on Certain Weighted Amalgam Spaces. J. Funct. Spaces 2020, 2020, 1-25. [CrossRef]

33. Feichtinger, H.G.; Feuto J. Pre-Dual of Fofana's Spaces. Mathematics 2019, 7, 528. [CrossRef]

34. Huang, L.; Liu, J.; Yang, D.; Yuan, W. Atomic and Littlewood-Paley characterizations of anisotropic mixed-norm Hardy spaces and their applications. J. Geom. Anal. 2019, 29, 1991-2067. [CrossRef]

35. Izuki, M.; Sawano, Y. Characterization of BMO via ball Banach function spaces, Vestn. St.-Peterbg. Univ. Mat. Mekh. Astron. 2017, $4,78-86$.

36. Duong, X.T.; Yan, L.X. On commutators of fractional integrals. Proc. Am. Math. Soc. 2004, 132, 3549-3557. [CrossRef]

37. Janson, S. Mean oscillation and commutators of singular integral operators. Ark. Math. 1978, 16, 263-270. [CrossRef] 